\title{
ON THE UNIFORM EXPONENTIAL STABILITY OF LINEAR TIME-DELAY SYSTEMS
}

\author{
M. DE LA SEN and NINGSU LUO
}

Received 24 September 2003

\begin{abstract}
This paper deals with the global uniform exponential stability independent of delay of timedelay linear and time-invariant systems subject to point and distributed delays for the initial conditions being continuous real functions except possibly on a set of zero measure of bounded discontinuities. It is assumed that the delay-free system as well as an auxiliary one are globally uniformly exponentially stable and globally uniform exponential stability independent of delay, respectively. The auxiliary system is, typically, part of the overall dynamics of the delayed system but not necessarily the isolated undelayed dynamics as usually assumed in the literature. Since there is a great freedom in setting such an auxiliary system, the obtained stability conditions are very useful in a wide class of practical applications.
\end{abstract}

2000 Mathematics Subject Classification: 93D10, 34C10, 93 C05.

1. Introduction. The stability and feedback stabilization of time-delay systems subject to constant point and distributed delays as well as time-varying ones has received important attention in the last years (see, e.g., [1, 2, 4, 5, 6, 8, 10, 11, 13]). A key point is that a system exhibiting stability in the absence of delays may lose that property for small delays and, in contrast, a stable delayed system may lose the property in the absence of delay (see, e.g., $[1,6,8]$ ). This paper deals with the global uniform exponential stability independent of delay (g.u.e.s.i.d.) of a class of homogeneous time-delay systems subject to combined point and distributed delays as well as integrodifferential Volterra-type delayed dynamics. The global stability is investigated for any function of initial conditions being everywhere continuous on its definition domain, a real interval $[-h, 0]$, where $h$ is the maximum delay in the system, except possibly on a set of zero measure where the function possesses bounded discontinuities. Necessary and sufficient global uniform stability conditions independent of delay are obtained if the delay-free system is globally uniformly exponentially stable (g.u.e.s.) and an auxiliary system is g.u.e.s.i.d. The obtained results are then applied to a number of particular cases of interest by setting different auxiliary systems including the standard delay-free one. The mathematical proofs are based on conditions which guarantee that a linear operator in a Banach space is compact within a domain that contains the closed complex right half-plane provided that another one defined for the auxiliary system is also compact within a (non necessarily identical) domain that contains the closed complex right half-plane. The auxiliary system may be a delay-free one or, in general, any particular parametrization of the whole system 
under study where part of the delayed dynamics is deleted. Some sufficient conditions for the system to be g.u.e.s. dependent on delay are also obtained by using the same mathematical outlines. Extensions are given for the case when the system is forced by impulsive inputs and also by considering the closed-loop stabilization of time-delay systems of the given class. The paper is organized as follows. Section 2 deals with the class of homogeneous delayed systems under study and with the definition of the auxiliary system. Section 3 is devoted to the main uniform stability result and the related ones for some particular auxiliary systems of interest. Some of those systems are defined by considering only delay-free dynamics or either pointdelayed, distributed-delayed, or even Volterra-type delayed dynamics together with a delay-free dynamics. Section 4 extends the above results to the presence of impulsive forcing functions. Section 5 is devoted to the stabilization of closed-loop systems of the given class under linear state or output-feedback controllers which can include delays. Some simple examples are discussed in Section 6 and, finally, conclusions end the paper.

Notation. (a) For the delayed system, $T:[0, \infty) \rightarrow L(X)$ is the inverse Laplace transform of the resolvent mapping $\hat{T}(s)$, which is holomorphic where it exists, with $X$ being the real Banach space of $n$-vector real functions endowed with the supremum norm on their definition domain. $\hat{T}^{-1}(s)$ takes the form $\left(\hat{T}_{J M}^{-1}(s)-\Delta \hat{T}_{J M}(s)\right)$, where $\hat{T}_{J M}(s)$ is defined similarly as $\hat{T}(s)$ for the auxiliary system, whose delay-free dynamics is defined by a square $n$-matrix $M$, and $\Delta \hat{T}_{J M}(s)=\hat{T}_{J M}^{-1}(s)-\hat{T}^{-1}(s)$. For all complex $s$ such that $\hat{T}_{J M}(s)$ exists, $\hat{T}(s)=\left(I-\hat{T}_{J M}(s) \Delta \hat{T}_{J M}(s)\right)^{-1} \hat{T}_{J M}(s)=\hat{\tilde{T}}_{J M}^{-1}(s) \hat{T}_{J M}(s)$.

The subindex $J=\left(J_{1}, J_{2}, J_{3}\right)$ denotes a triple for sets of indices referred to as the particular subsets of real constants describing point delays $\left(J_{1}\right)$, infinitely distributed Volterra-type delays $\left(J_{2}\right)$, and finitely distributed delays $\left(J_{3}\right)$ of the system which are also present in the auxiliary system. For instance, $1 \in J_{1} \Rightarrow h_{1}>0$ is a point delay of the time-delay system which is also present in the auxiliary system, and so on. Also, $\operatorname{Card}\left(J_{1}\right) \leq m$, $\operatorname{Card}\left(J_{2}\right) \leq m^{\prime}+1, \operatorname{Card}\left(J_{3}\right) \leq m^{\prime \prime}$. If a pure convolution Volterra-type dynamics $\int_{0}^{t} d \alpha_{0}(\tau) A_{\alpha_{0}} x(t-\tau)$ is present, then it is described by a fictitious delay $h_{0}^{\prime}=$ 0 . If such a term is not present, then $\operatorname{Card}\left(J_{2}\right) \leq m^{\prime}$. The remaining infinitely distributed delays give contributions $\int_{0}^{t} d \alpha_{i}(\tau) A_{\alpha_{i}} x\left(t-\tau-h_{i}^{\prime}\right)$, with finite real constants $h_{i}^{\prime}>0$ with $i=1,2, \ldots, m^{\prime}$, to $\dot{x}(t)$ which are point delays under the integral symbol. It is said that the delays are infinitely distributed because the contribution of the delayed dynamics is made under an integral over $[0, \infty)$ as $t \rightarrow \infty$, that is, $x\left(t-\tau-h_{i}^{\prime}\right)$ acts on the dynamics of $x(t)$ from $\tau=0$ to $\tau=t$ for finite $t$ and as $t \rightarrow \infty$.

(b) $\hat{T}^{\prime-1}(s, \varphi)=\hat{T}_{J M}^{\prime-1}(s, \varphi)-\Delta \hat{T}_{J M}^{\prime}(s, \varphi)$ is a complex operator-valued function with domain in $\mathbb{C} \times[-\pi, \pi]^{m+m^{\prime}+m^{\prime \prime}+1} \subset \mathbb{C} \times \mathbb{R}^{m+m^{\prime}+1}$ with $[-\pi, \pi]^{m+m^{\prime}+m^{\prime \prime}+1}$ being the cross product of $[-\pi, \pi]$ by itself $\left(m+m^{\prime}+m^{\prime \prime}+1\right)$ times, $\varphi^{T}=\left(\varphi_{1}, \varphi_{2}, \ldots\right.$, $\left.\varphi_{m+m^{\prime}+m^{\prime \prime}+1}\right), \varphi_{m+1}=0$ (since $h_{0}^{\prime}=0$ ), and range in $\mathbb{C}^{n}$.

(c) $N(s, \hat{h})=N_{J M}(s, \hat{h})\left[I-N_{J M}^{-1}(s, \hat{h}) \Delta N_{J M}(s, \hat{h})\right]=N_{J M}(s, \hat{h}) \cdot \tilde{N}_{J M}(s, \hat{h})$ is an operator-valued function with domain in $\mathbb{C} \times \mathbb{R}^{m+m^{\prime}+m^{\prime \prime}+1}$, where $\hat{h}=\left(\hat{h}_{1}^{\prime T}, \hat{h}_{2}^{\prime T}\right)^{T}$ and $h_{0}^{\prime}=0$ for $s=j \omega$, any $\hat{h}$ with $\varphi_{m+1}=0$, and remaining components $\varphi_{i}$ in $[-\pi, \pi]$ whose values depend on $h_{i}(i \leq m)$ or $h_{i}^{\prime}\left(i \geq m^{\prime}+2\right)$. Similarly, 


$$
\begin{aligned}
N_{J M}(j \omega, \hat{h}) & =\hat{T}_{J M}^{\prime-1}(j \omega, \varphi)=\hat{T}_{J M}^{\prime-1}(j \omega), \\
\Delta N_{J M}(j \omega, \hat{h}) & =\Delta \hat{T}_{J M}^{\prime}(j \omega, \varphi)=\Delta \hat{T}_{J M}(j \omega), \\
\tilde{N}_{J M}(j \omega, \hat{h}) & =\hat{\tilde{T}}_{J M}^{\prime-1}(j \omega, \varphi)=\hat{\tilde{T}}_{J M}^{\prime-1}(j \omega), \\
\tilde{N}(j \omega, \hat{h}) & =\hat{\tilde{T}}^{\prime-1}(j \omega, \varphi)=\hat{\tilde{T}}^{-1}(j \omega),
\end{aligned}
$$

for the above $\hat{h}$ and $\varphi$.

Note that $\hat{T}, \hat{T}^{\prime}$, and $N^{-1}$ are distinct mathematical objects but, however, they take identical values for all pure imaginary $s=j \omega$ and a corresponding $\varphi_{i} \in[-\pi, \pi]$ such that $e^{-j \omega_{i}}=e^{ \pm j \varphi_{i}}$ with $\varphi_{m+1}=h_{0}^{\prime}=0$. The same applies to the related objects referred to as the auxiliary system.

2. Problem statement. Consider the following linear and time-invariant system subject to point and distributed dynamics and to an impulsive function:

$$
\begin{aligned}
\dot{x}(t)= & \sum_{i=0}^{n} A_{i} x\left(t-h_{i}\right)+\sum_{i=0}^{m^{\prime}} \int_{0}^{t} d \alpha_{i}(\tau) A_{\alpha_{i}} x\left(t-\tau-h_{i}^{\prime}\right) \\
& +\sum_{i=m^{\prime}+1}^{m^{\prime}+m^{\prime \prime}} \int_{t-h_{i}^{\prime}}^{t} d \alpha_{i}(t-\tau) A_{\alpha_{i}} x(\tau)+\sum_{i \in \mathbf{I}} b_{i} \delta\left(t-t_{i}\right),
\end{aligned}
$$

where $A_{0}$ and $A_{i}, A_{\alpha_{k}}\left(i=1,2, \ldots, m ; k=0,1, \ldots, m^{\prime}+m^{\prime \prime}\right)$ belong to the spaces of unbounded and bounded operators, respectively, on a Banach space of $n$-vector real functions $x \in \mathbf{X}$ endowed with the supremum norm where the vectors of point and distributed constant delays are $\hat{h}=\left(0, h_{1}, h_{2}, \ldots, h_{m}\right)$ and $\hat{h}^{\prime}=\left(\hat{h}_{1}^{\prime T} \vdots \hat{h}_{2}^{\prime T}\right)^{T}=\left(0, h_{1}^{\prime}, h_{2}^{\prime}, \ldots\right.$, $\left.h_{m^{\prime}}^{\prime} \vdots h_{m^{\prime}+1}^{\prime}, h_{m^{\prime}+2}^{\prime}, \ldots, h_{m^{\prime}+m^{\prime \prime}}^{\prime}\right)^{T}$, respectively, with $h_{i} \geq 0$ and $h_{k}^{\prime} \geq 0\left(i=1,2, \ldots, m^{\prime}+\right.$ $\left.m^{\prime \prime}\right)$ and $h_{0}=h_{0}^{\prime}=0, A_{0} \equiv A, A_{\alpha_{0}} \equiv A_{\alpha}$, and $\alpha_{0}(\cdot) \equiv \alpha(\cdot)$. The functions $\alpha_{i}:[0, \infty) \rightarrow \mathbb{R}$ and $\alpha_{k}:\left[0, h_{k}^{\prime}\right] \rightarrow \mathbb{R}$ are continuously differentiable real functions within their definition domains except possibly on sets of zero measure where the time-derivatives have bounded discontinuities. All or some of the $\alpha_{i}(\cdot)$ and $\alpha_{k}(\cdot)$ may be alternatively matrix functions, $\alpha_{i}:[0, t] \rightarrow \mathbb{R}^{n \times n}$ for $t \in \mathbb{R}_{+}$and $\alpha_{i}:\left[0, h_{k}^{\prime}\right] \rightarrow \mathbb{R}^{n \times n}$. We will not make any explicit difference between both possibilities in the notation for the sake of simplicity. The impulsive input $v(t)=\sum_{i \in \mathrm{I}} b_{i} \delta\left(t-t_{i}\right)$ is built with the finite or infinite sequence of Dirac impulses $\delta\left(t-t_{i}\right)$ at the sequence of time instants $\left\{t_{i} ; i \in \mathbf{I}\right\}$ with $t_{i+1}>t_{i}$ for some totally ordered proper or improper numerable subset $\mathbf{I} \subseteq \mathbb{N}$. If $\operatorname{Card}(\mathbf{I})=p<\infty$, then $v(t):=\sum_{i=1}^{p} b_{i} \delta\left(t-t_{i}\right)$ and $\mathbf{I}:=\{i \in \mathbb{N}: i \leq p\}$. Note that system (2.1) is very general since it includes point-delayed dynamics like, for instance, in typical war/peace models or the so-called Minorski's problem appearing when controlling the lateral dynamics of a ship. It also includes real constants $h_{i}^{\prime}\left(i=0,1, \ldots, m^{\prime}\right)$, with $h_{0}^{\prime}=0$, associated with infinitely distributed delayed contributions to the dynamics through integrals, related to the $\alpha_{i}(\cdot), i=0,1, \ldots, m^{\prime}$. Such delays are relevant, for instance, in viscoelastic fluids, electrodynamics, and population growth $[1,4,6]$. In particular, an integrodifferential 
Volterra-type term is also included through $h_{0}^{\prime}=0$. Apart from those delays, the action of finite distributed delays characterized by real constants $h_{i}^{\prime}\left(i=0,1, \ldots, m^{\prime}+m^{\prime \prime}\right)$ is also included in (2.1). That kind of delays is well known, for instance, in econometric models related to production rate [4]. Finally, the impulsive input $v(t)=\sum_{i \in \mathrm{I}} b_{i} \delta\left(t-t_{i}\right)$ generates bounded discontinuities of the solution trajectory $x(t)$ at $t=t_{i}(i \in \mathbf{I})$, see, for instance, $[10,11,12]$. The following technical hypothesis are made.

(H1) All the operators $A_{k}(0 \leq k \leq m), A_{\alpha_{k}}\left(0 \leq k \leq m^{\prime}+m^{\prime \prime}\right)$ are in $L(X):=L(X, X)$, the set of linear operators on $X$ of dual $X^{*}$, and $h_{k}$ and $h_{\ell}^{\prime}(k=1,2, \ldots, m ; \ell=$ $\left.0,1, \ldots, m^{\prime}+m^{\prime \prime}\right)$ are nonnegative constants with $h_{0}=h_{0}^{\prime}=0$ and $h=$ $\operatorname{Max}\left(\operatorname{Max}_{1 \leq i \leq n}\left(h_{i}\right), \operatorname{Max}_{1 \leq i \leq m^{\prime}+m^{\prime \prime}}\left(h_{i}^{\prime}\right)\right)$.

(H2) The initial conditions of (2.1) are real $n$-vector functions $\phi \in C(h)$, where $C_{e}(h):=\left\{\phi=\phi_{1}+\phi_{2}: \phi_{1} \in C(h), \phi_{2} \in B^{0}(h)\right\}$ with $C(h):=\left\{C^{0}([-h, 0] ; X)\right\} ;$ that is, the set of continuous mappings from $[-h, 0]$ into the Banach space $X$ with norm $\bar{\phi}:=|\phi|=\operatorname{Sup}\{\|\phi(t)\|:-h \leq t \leq 0\},\|\cdot\|$ denoting the Euclidean norm of vectors in $\mathbb{R}^{n}$ and matrices in $\mathbb{R}^{n \times n}$ and $B^{0}(h):=\{\phi:[-h, 0] \rightarrow X\}$, is the set of real bounded vector functions on $X$ endowed with the supremum norm having support of zero measure. Roughly speaking, $\phi \in B^{0}(h)$ if and only if it is almost everywhere zero except at isolated points within $[-h, 0]$ where it is bounded. Thus, $\phi \in C_{e}(h)$ if and only if it is almost everywhere continuous in $[-h, 0]$ except possibly on a set of zero measure of bounded discontinuities. $C_{e}(h)$ is also endowed with the supremum norm since $\phi=\phi_{1}+\phi_{2}$, some $\phi_{1} \in C(h)$, $\phi_{2} \in B^{0}(h)$ for each $\phi \in C_{e}(h)$. In the following, the supremum norms on $L(X)$ are also denoted with $|\cdot|$.

(H3) The linear operators $A_{\alpha_{i}} \in L(X)$, with abbreviated notation $A_{\alpha_{0}}=A_{\alpha}$, are closed and densely defined linear operators with respective domain and range $D\left(A_{\alpha_{i}}\right)$ and $R\left(A_{\alpha_{i}}\right) \subset X\left(i=0,1, \ldots, m^{\prime}+m^{\prime \prime}\right)$. The functions $\alpha_{i}$ belong to $C^{0}([0, \infty) ; \mathbb{R}) \cap$ $B V_{\text {loc }}\left(\mathbb{R}_{+}\right)\left(i=0,1, \ldots, m^{\prime}\right)$ and $C^{0}([-h, 0) ; \mathbb{R})\left(i=0,1, \ldots, m^{\prime}+m^{\prime \prime}\right)$ being everywhere differentiable with possibly bounded discontinuities on subsets of zero measure of their definition domains with $\int_{0}^{\infty} e^{v t}\left|d \alpha_{i}(t)\right|<\infty$ and some nonnegative real constant $v\left(i=0,1, \ldots, m^{\prime}\right)$. If $\alpha_{i}(\cdot)$ is a matrix function $\alpha_{i}:[0, \infty) \times X^{*}$ $\rightarrow L\left(X, X^{*}\right)$, then it is in $C^{0}\left([0, \infty) ; \mathbb{R}^{n \times n}\right) \cap B V_{\text {loc }}\left(\mathbb{R}_{+}^{n \times n}\right)$ with $\int_{0}^{\infty} e^{v t}\left|d \alpha_{i}(t)\right|<\infty$ and its entries being everywhere time-differentiable with possibly bounded discontinuities on a subset of zero measure of their definition domains.

The integrability of the $\alpha_{i}(\cdot)$-functions (or matrix functions) on $\left[t-h_{i}^{\prime}, t\right], m^{\prime}+1 \leq$ $i \leq m^{\prime}+m^{\prime \prime}$, follows since their definition domain is bounded. The above technical hypotheses (H1), (H2), and (H3) guarantee the existence and uniqueness of the solution of the homogeneous system (2.1) (i.e., $v \equiv 0$ ) for each initial condition $\phi \in C_{e}(h)$. Take Laplace transforms in (2.1) by using the convolution theorem and the relations $d \alpha(\tau)=\dot{\alpha}(\tau) d \tau$. It follows that $d \hat{\alpha}_{i}(s)=s \hat{\alpha}_{i}(s)-\alpha_{i}(0)$, where $\hat{f}(s)$ denotes the Laplace transform of $f(t)$. Thus, one gets from (2.1)

$$
\hat{x}(s)=\hat{T}(s)\left(x\left(0^{+}\right)+\sum_{i \in \mathbf{I}} b_{i} e^{-t_{i} s}\right),
$$


where

$$
\begin{aligned}
\hat{T}(s)= & {\left[s\left(I-\hat{\alpha}(s) A_{\alpha}-\sum_{i=1}^{m^{\prime}} \hat{\alpha}_{i}(s) A_{\alpha_{i}} e^{-h_{i}^{\prime} s}-\sum_{i=m^{\prime}+1}^{m^{\prime}+m^{\prime \prime}} \hat{\alpha}_{i}(s) A_{\alpha_{i}}\left(1-e^{-h_{i}^{\prime} s}\right)\right)\right.} \\
& \left.-A-\sum_{i=1}^{m} A_{i} e^{-h_{i} s} \alpha(0) A_{\alpha}+\sum_{i=1}^{m^{\prime}} \alpha_{i}(0) A_{\alpha_{i}} e^{-h_{i}^{\prime} s}+\sum_{i=m^{\prime}+1}^{m^{\prime}+m^{\prime \prime}} \alpha_{i}(0) A_{\alpha_{i}}\left(1-e^{-h_{i}^{\prime} s}\right)\right]^{-1} .
\end{aligned}
$$

Note that (2.1) is guaranteed to be g.u.e.s.i.d. if and only if $\hat{T}(s)$ exists within some region including properly the right complex plane, in other words, if it is compact for Re $s>-\alpha_{0}$, for some constant $\alpha_{0} \in \mathbb{R}_{+}$, since then all the entries of its Laplace transform $T(t)$ decay with exponential rate on $[0, \infty)$ for $\phi \in C_{e}(h)$, and then $|x(t)|$ decays with exponential rate on $\mathbb{R}_{+}$. The unique solution of the homogeneous system (2.1) for each $\phi \in C_{e}(h)$ may be equivalently written in infinitely many cases by first rewriting (2.1) by considering different "auxiliary" reference homogeneous systems plus additional terms considered as forcing actions. The next arrangements lead to conditions guaranteeing that the homogeneous system (2.1) is g.u.e.s.i.d. if it is g.u.e.s. in the absence of delay (i.e., for $h=0$ ). Through this arrangement, it is not necessarily requested for $\dot{z}(t)=$ $A z(t)$, which is in fact one of the possible auxiliary homogeneous systems for (2.1), to be g.u.e.s.i.d. for any $\phi \in C_{e}(h)$. Thus, note that (2.1) may be compactly written as

$$
\dot{x}(t)=L x_{t}+v(t)=L_{J M} x_{t}+\left(\bar{L}_{J M} x_{t}+v(t)\right),
$$

where $L=L_{J M}+\bar{L}_{J M}$ is a linear operator in $L(X)$ defined by $L x_{t}$ equalized by the unforced right-hand side of (2.1), where $x_{t}$ denotes the string $x:[t-h, t] \rightarrow X$ of the solution to (2.1) for $\phi \in C_{e}(h)$ for all $t \geq 0$, and $L_{J M}$ and $\bar{L}_{J M}$ are also linear operators in $L(X)$ which define a nonunique additive decomposition of $L$ that depends on $M$, an $n$-square arbitrary real matrix, and $J$, a triple $J=\left(J_{1}, J_{2}, J_{3}\right)$ of indices $J_{i}(i=1,2,3)$. The $M$-matrix and the $J$-triple define the subsequent g.u.e.s.i.d. auxiliary system. That property is the starting point to derive conditions for the current delayed system (2.1) to be g.u.e.s.i.d. as well. The auxiliary system is

$$
\begin{aligned}
\dot{z}(t)= & L_{J M} z_{t} \\
= & M z(t)+(A-M) z(t)+\sum_{i \in J_{1}} A_{i} x\left(t-h_{i}\right) \\
& +\sum_{i \in J_{2}} \int_{0}^{t} d \alpha_{i}(\tau) A_{\alpha} x\left(t-h_{i}^{\prime}-\tau\right)+\sum_{i \in J_{3}} \int_{t-h_{i}^{\prime}}^{t} d \alpha_{i}(t-\tau) A_{\alpha_{i}} x(\tau)
\end{aligned}
$$


subject to initial conditions $z(t)=\phi(t)$ for $t \in[-h, 0]$ with $\phi \in C_{e}(h)$, some given matrix $M \in \mathbb{R}^{n \times n}$, and

$$
\begin{aligned}
& J_{1}=\{\left\{i \in \mathbb{N}: 1 \leq i \leq m, h_{i} \text { is a point delay in } \dot{z}(t)=L_{J M} z_{t}\right\}, \\
& J_{2}=\left\{i \in \mathbb{N}: 0 \leq i \leq m^{\prime}, h_{i}^{\prime}\right. \text { is a constant defining an infinitely } \\
&\text { distributed delay in } \left.\dot{z}(t)=L_{J M} z_{t}\right\}, \\
& J_{3}=\left\{i \in \mathbb{N}: m^{\prime}+1 \leq i \leq m^{\prime}+m^{\prime \prime}, h_{i}^{\prime}\right. \text { is a finitely }\text { distributed delay in } \left.\dot{z}(t)=L_{J M} z_{t}\right\}
\end{aligned}
$$

are respective proper or improper subsets of $N_{1}=\{1,2, \ldots, m\}, N_{2}=\left\{0,1, \ldots, m^{\prime}\right\}$, and $N_{3}=\left\{m^{\prime}+1, m^{\prime}+2, \ldots, m^{\prime}+m^{\prime \prime}\right\}$ that define the $J$-triple. $\bar{J}_{i}=N_{i} / J_{i}$ denotes the complement of $J_{i}$ in $N_{i}(i=1,2,3)$. Then $i \in J_{1}$ if and only if the point delay $h_{i}$ is explicit in the auxiliary system (2.5), and $i \in J_{2,3}$ if and only if the distributed delay $h_{i}^{\prime}$ is explicit in (2.5). In particular, $J_{i}=\varnothing$ (the empty set) for some $i \in\{1,2,3\}$ if there is no delay of the corresponding class in (2.5). Thus, (2.1) may be compactly rewritten as

$$
\dot{x}(t)=L x_{t}+v(t)=L_{J M} x_{t}+\left(\bar{L}_{J M} x_{t}+v(t)\right)
$$

with $x(t)=\phi(t)$ for $t \in[-h, 0), \phi \in C_{e}(h)$, where

$$
\begin{aligned}
L x_{t}= & A x(t)+\sum_{i=1}^{m} A_{i} x\left(t-h_{i}\right) \\
& +\sum_{i=0}^{m^{\prime}} \int_{0}^{t} d \alpha_{i}(\tau) A_{\alpha_{i}} x\left(t-\tau-h_{i}^{\prime}\right) \sum_{i=m^{\prime}+1}^{m^{\prime}+m^{\prime \prime}} \int_{t-h_{i}^{\prime}}^{t} d_{\alpha_{i}}(\tau) A_{\alpha_{i}} x(\tau), \\
L_{J M} x_{t}= & M x(t)+\sum_{i \in J_{1}} A_{i} x\left(t-h_{i}\right)+\sum_{i \in J_{2}} \int_{0}^{t} d \alpha_{i}(\tau) A_{\alpha_{i}} x\left(t-\tau-h_{i}^{\prime}\right) \\
& \times \sum_{i \in J_{3}} \int_{t-h_{i}^{\prime}}^{t} d \alpha_{i}(t-\tau) A_{\alpha_{i}} x(\tau), \quad t \geq 0, \\
\bar{L}_{J M} x_{t} \equiv & \left(L-L_{J M}\right) x_{t} \\
= & (M-A) x_{t}+\sum_{i \in \bar{J}_{1}} A_{i} x\left(t-h_{i}\right)+\sum_{i \in \bar{J}_{2}} \int_{0}^{t} d \alpha_{i}(\tau) A_{\alpha_{i}} x\left(t-\tau-h_{i}^{\prime}\right) \\
& \times \sum_{i \in \bar{J}_{3}} \int_{t-h_{i}^{\prime}}^{t} d \alpha_{i}(t-\tau) A_{\alpha_{i}} x(\tau), \quad t \geq 0 .
\end{aligned}
$$


In view of (2.7), the unique solution of (2.1) for any $\phi \in C_{e}(h)$ is

$$
\begin{aligned}
x(t, \phi)= & T(t) \phi\left(0^{+}\right)+\sum_{i=1}^{m} \int_{-h_{i}}^{0} T(t-\tau) \phi(\tau) d \tau \\
& +\sum_{i=1}^{m^{\prime}+m^{\prime \prime}} \int_{-h_{i}^{\prime}}^{0} T(t-\tau) \phi(\tau) d \tau+\sum_{i \in \mathbf{I}} T\left(t-t_{i}\right) b_{i} U\left(t-t_{i}\right) \\
= & T_{J M}(t) \phi\left(0^{+}\right)+\sum_{i \in J_{1}} \int_{-h_{i}}^{0} T_{J M}(t-\tau) \phi(\tau) d \tau+\sum_{i \in J_{2} \cup J_{3}} \int_{-h_{i}^{\prime}}^{0} T_{J M}(t-\tau) \phi(\tau) d \tau \\
& \times \int_{0}^{t} T_{J M}(t-\tau)\left[(A-M) x(\tau)+\sum_{i \in \bar{J}_{1}} A_{i} x\left(\tau-h_{i}\right)\right. \\
& \quad+\sum_{i \in J_{2}} \int_{0}^{\tau} T_{J M}\left(\tau^{\prime}\right) \dot{\alpha}_{i}\left(\tau^{\prime}\right) A_{\alpha_{i}} x\left(\tau-\tau^{\prime}-h_{i}^{\prime}\right) d \tau^{\prime} \\
& \left.\times \sum_{i \in J_{3}} \int_{\tau-h_{i}^{\prime}}^{\tau} T_{J M}\left(\tau-\tau^{\prime}\right) \dot{\alpha}_{i}\left(\tau-\tau^{\prime}\right) A_{\alpha_{i}} x\left(\tau^{\prime}\right) d \tau^{\prime}\right] d \tau \\
& +\sum_{i \in \mathbf{I}} T\left(t-t_{i}\right) b_{i} U\left(t-t_{i}\right),
\end{aligned}
$$

where $T(t)$ satisfies $\dot{T}(t)=L T_{t}$ for $t>0$ with $T(0)=I$ (the $n$-identity matrix) and $T(t)=0$ for $t<0$ with $T(t)$ being the inverse Laplace transform of $\hat{T}^{-1}(s), \hat{T}(s)$ defined in (2.3), and $T_{J M}(t)$ satisfies $\dot{T}_{J M}(t)=L_{J M}\left(T_{J M}\right)_{t}$ for $t>0$ with $T_{J M}(0)=I$ and $T_{J M}(t)=0$ for $t<0 . U(t)=1(t)$ is the unity Heaviside function. Thus, $T_{J M}(t)$ is the inverse Laplace transform of the holomorphic (where it exists) mapping $\hat{T}_{J M}(s)$ with

$$
\begin{aligned}
\hat{T}_{J M}(s)= & {\left[s\left(I-\sum_{i \in J_{2}} \hat{\alpha}_{i}(s) A_{\alpha_{i}} e^{-h_{i}^{\prime} s}-\sum_{i \in J_{3}} \hat{\alpha}_{i}(s) A_{\alpha_{i}}\left(1-e^{-h_{i}^{\prime} s}\right)\right)-M-\sum_{i \in J_{1}} A_{i} e^{-h_{i} s}\right.} \\
& \left.\times \sum_{i \in J_{2}} \alpha_{i}\left(0^{+}\right) A_{\alpha_{i}}+\sum_{i \in J_{3}} \alpha_{i}\left(0^{+}\right) A_{\alpha_{i}}\left(1-e^{-h_{i}^{\prime} s}\right)\right]^{-1} .
\end{aligned}
$$

Note that $T(t) \quad\left(\equiv T_{J M}(t)\right.$ if $J=\left(N_{1}, N_{2}, N_{3}\right)$ ) and $T_{J M}(t)$ for any $J$-triple are $C_{0^{-}}$ semigroups on $C_{e}(h)$ of operators of $L(X)$. In particular, if $J_{i}=\varnothing(i=1,2,3)$, then $L_{J M} z_{t}=M z(t), T_{J M}(t)=e^{A t}$ is an analytic semigroup if $J_{1}$ and $J_{3}$ are empty, and $J_{2}=\{0\}$ (i.e., $h_{0}^{\prime}=0$ ) is the unique contribution to a Volterra-type integral term, then $L_{J M} z_{t}=M z(t)+\int_{0}^{t} d \alpha(\tau) A_{\alpha} x(t-\tau)$ and $T_{J M}(t)$ is a transition operator if $\hat{T}_{J M}(s)$ is compact for $\operatorname{Re} s>-\gamma_{J M}\left(\gamma_{J M} \in \mathbb{R}_{+}\right)$.

REMARK 2.1. Note that the compactness of the operator-valued functions $\hat{T}(s)$ and $\hat{T}_{J M}(s)$ for all Re $s>-\gamma$ and Re $s>-\gamma_{J M}$, some $\gamma \in \mathbb{R}_{+}$and $\gamma_{J M} \in \mathbb{R}_{+}$, respectively, if $\dot{x}(t)=L x_{t}, \dot{z}(t)=L z_{t}$, respectively, are g.u.e.s.i.d for all $\phi \in C_{e}(h)$, holds directly if they are bounded provided that $X$ is considered as a Hilbert space endowed with the usual inner product norm. The stability properties of the operator-valued function $T:[0, \infty) \rightarrow L(X)$ are independent of the use of any of both alternative formal 
characterizations. Thus, if $X$ is a Hilbert space, then there exist dense injective mappings $X \rightarrow X^{*}$ (dual of $\left.X\right) \rightarrow X^{* *}$ (dual of $\left.X^{*}\right) \equiv X$, instead of the generic result which may include in some cases proper inclusion $X^{* *} \supset X \neq X^{* *}$ so that $X$ is a reflexive linear space and any operator in $L\left(X^{* *}, X\right)(\equiv L(X, X)=L(X)$ in this case) is compact if and only if it is completely continuous (i.e., if it maps any weakly convergent sequence into a strongly convergent one with respect to the norm topology). Thus, $\hat{T}(s)$ is compact (or completely continuous) where it exists since $(\hat{T})^{*} \cdot \hat{T}$ is bounded for $\operatorname{Re} s>-\gamma$. The same property holds for any $\hat{T}_{J M}$ for $\operatorname{Re} s>-\gamma_{J M}$.

Note that $\hat{T}(s)=\left[\hat{T}_{J M}(s)-\Delta \hat{T}_{J M}(s)\right]^{-1}=\hat{T}_{J M}(s)\left[I-\hat{T}_{J M}^{-1}(s) \Delta \hat{T}_{J M}(s)\right]^{-1}$ in the definition domain of $\hat{T}_{J M}$ for any auxiliary system defined from some given $J$-triple. The following special cases are of interest.

SPECIAL CASES. (1) The auxiliary system is delay-free: $J=\left(J_{1}, J_{2}, J_{3}\right)$ with $J_{i}=\varnothing$ $(i=1,2,3)$ so that the auxiliary system is $\dot{z}(t)=M z(t)$. This is the case usually treated in the literature (see, e.g., $[4,6])$. Thus, $\bar{J}_{i}=N_{i}(i=1,2,3)$ and $T_{J M}(t)=e^{M t}$ is an analytic semigroup.

(2) The auxiliary system is subject to delay-free and all point delays: $J_{1}=N_{1}$ and $J_{2} \cup J_{3}=\varnothing$ so that $\bar{J}_{1}=\varnothing$ and $\bar{J}_{i}=N_{i}(i=2,3)$. Then, $\dot{z}(t)=M z(t)+\sum_{i=1}^{m} A_{i} z(t-$ $h_{i}$ ) with initial conditions $z(t)=\phi(t), \phi \in C_{e}(h)$, for $t \in\left[\max _{1 \leq i \leq 0}\left(-h_{i}\right), 0\right]$ so that $\dot{T}_{J M}(t)=M T_{J M}(t)+\sum_{i=1}^{m} A_{i} T_{J M}\left(t-h_{i}\right)$ with $T_{J M}(0)=I$ and $T_{J M}(t)=0(t<0)$ yields a unique solution $T_{J M}(t)=e^{M t}\left(I+\sum_{i=1}^{m} \int_{h_{i}}^{t} e^{-M \tau} A_{i} T_{J M}\left(\tau-h_{i}\right) d \tau\right)$ for $t \geq 0$.

(3) The auxiliary system is subject to delay-free dynamics and Volterra-integraltype dynamics: $J_{1} \cup J_{3}=\varnothing, \bar{J}_{i}=N_{i}$, and $\bar{J}_{2}=\left\{1,2, \ldots, m^{\prime}\right\}$. Thus, $\dot{z}(t)=M z(t)+$ $\int_{0}^{t} d \alpha(\tau) A_{\alpha} z(t-\tau)$. In particular, $T_{J M}(t)$ is ensured to be a transition operator with $\left|T_{J M}(t)\right| \leq K e^{-\rho t}$, for some positive real constants $K$ and $\rho$ and all $t \geq 0$ (see, e.g., [1, 9]) if

$$
\hat{T}_{J M}^{-1}(s)=\left[s\left(I-\hat{\alpha}(s) A_{\alpha}\right)+\alpha(0) A_{\alpha}-M\right]^{-1}
$$

is compact for Re $s>-\rho$, any real constant $\rho<\gamma_{J M}$, and $\left|d^{i}\left(\hat{T}_{J M}^{-1}(s)\right) / d s^{i}\right|<\mid K /(s+$ $p)^{i-1} \mid($ for $i=1,2,3$ ).

(4) The auxiliary system has delay-free dynamics and all the infinitely distributed delays: now, $J=\left(J_{1}, J_{2}, J_{3}\right)$ with $J_{i}=\varnothing(i=1,3)$ and $J_{2}=N_{2}$ so that $\bar{J}_{i}=N_{i}(i=1,3)$ and $\bar{J}_{2}=\varnothing$, what leads to

$$
\dot{z}(t)=M z(t)+\sum_{i=0}^{m^{\prime}} \int_{0}^{t} d \alpha_{i}(\tau) z\left(t-\tau-h_{i}^{\prime}\right)
$$

under initial conditions $\phi \in C_{e}(h)$. Thus, one gets

$$
\dot{T}_{J M}(t)=M T_{J M}(t)+\sum_{i=0}^{m} \int_{0}^{t} d \alpha_{i}(\tau) A_{\alpha_{i}} T_{J M}\left(t-\tau-h_{i}^{\prime}\right)
$$


for $t>0$ with $T_{J M}(0)=I, T_{J M}(t)=0$ for $t<0$, whose unique solution for all $t>0$ is

$$
T_{J M}(t)=e^{M t}\left(I+\sum_{i=0}^{m^{\prime}} \int_{0}^{t} \int_{0}^{\tau} e^{-M \tau} d \alpha_{i}(\tau) A_{\alpha_{i}} T_{J M}\left(\tau-\tau^{\prime}-h_{i}^{\prime}\right) d \tau^{\prime}\right) .
$$

(5) The auxiliary system has delay-free dynamics and all the finitely distributed delays: now, $J=\left(J_{1}, J_{2}, J_{3}\right)$ with $J_{i}=\varnothing(i=1,2)$ and $J_{3}=N_{3}$ so that $\bar{J}_{i}=N_{i}(i=1,2)$ and $\bar{J}_{3}=\varnothing$. Under the same initial conditions as in the above case, one gets $\dot{z}(t)=$ $M z(t)+\sum_{i=m^{\prime}+1}^{m^{\prime}+m_{t-h_{i}^{\prime}}^{\prime}} d \alpha_{i}(t-\tau) A_{\alpha_{i}} z(\tau)$ for $t>0$, which is also satisfied by the transition operator of the auxiliary system whose unique solution under the same initial conditions as in case (4) is

$$
T_{J M}(t)=e^{M t}\left(I+\sum_{i=m^{\prime}+1}^{m^{\prime}+m^{\prime \prime}} \int_{0}^{t} \int_{\tau-h_{i}^{\prime}}^{\tau} e^{-M \tau^{\prime}} d \alpha_{i}\left(\tau-\tau^{\prime}\right) A_{\alpha_{i}} T_{J M}\left(\tau^{\prime}\right) d \tau^{\prime}\right) .
$$

\section{Uniform stability of the homogeneous system}

THEOREM 3.1. Assume that (2.1) is g.u.e.s. for $\hat{h}=0$ and that $\dot{z}(t)=L_{J M} z_{t}$ is g.u.e.s.i.d. for all $\phi \in C_{e}(h)$. Thus, the homogeneous equation (2.1), $\dot{x}(t)=L_{J M} x_{t}$, is g.u.e.s.i.d. (i.e., for all $\left.\phi \in C_{e}(h)\right)$ if and only if the operator-valued function

$$
\hat{\tilde{T}}_{J M}^{\prime-1}(j \omega, \varphi)=\left(I-\hat{T}_{J M}^{\prime}(j \omega, \varphi) \Delta \hat{T}_{J M}^{\prime}(j \omega, \varphi)\right)^{-1}
$$

exists for all real $\omega \in(0, \infty)$ and all $\varphi_{i k_{i}} \in[-\pi, \pi](i=1,2,3)\left(\varphi_{21}=0\right) ; k_{i}=1,2, \ldots, p$ with $p=m$ if $i=1, p=m^{\prime}+1$ if $i=2$, and $p=m^{\prime \prime}$ if $i=3$.

Proof. First note that the argument $\omega=0$ for the above operator-valued function is excluded from the conditions since (2.1) is g.u.e.s. for $\hat{h}=0$. System (2.1) is g.u.e.s.i.d. if and only if $N^{-1}(s, \hat{h})$ exists for Re $s>-\gamma$ (some $\gamma \in \mathbb{R}_{+}$) for any sets of delays. Since $N_{J M}^{-1}(s)(s, \hat{h}) N(s, \hat{h})=\left[I-N_{J M}^{-1}(s, \hat{h}) \Delta N_{J M}(s, \hat{h})\right]$ and $N_{J M}(s, \hat{h})$ has an inverse $\operatorname{Re} s>$ $-\gamma_{J M}$ (some $\gamma_{J M} \in \mathbb{R}_{+}$) for all the sets of delays explicit in the auxiliary system (2.5), $N^{-1}(s, \hat{h})$ exists for a pair $(s, \hat{h})$ if and only if $\tilde{N}^{-1}(s, \hat{h})$ exists for $(s, \hat{h})$, where

$$
\tilde{N}_{J M}(s, \hat{h})=N_{J M}^{-1}(s, \hat{h}) N(s, \hat{h})=\left[I-N_{J M}^{-1}(s, \hat{h}) \Delta N_{J M}(s, \hat{h})\right] .
$$

Proof OF NECESSITY. The rank condition cannot fail for $\omega=0$ since system (2.1) is g.u.e.s. and then $N(j 0, \hat{h})$ is full rank for any set of delays. Assume that $\operatorname{rank}\left[\hat{\tilde{T}}_{J M}^{\prime}(j \omega, \varphi)\right]<n$ for some $\omega \neq 0$, then $\operatorname{rank}\left[\tilde{N}_{J M}(j \omega, \hat{h})\right]<n$ and the set of delays $h_{i}=\varphi_{i} / \omega$ is the $i$ th component of $\varphi$. This is a contradiction and necessity follows.

Proof OF SUFFICIENCY. Since $\hat{T}(s), \hat{T}_{J M}(s)$ (and $\left.N^{-1}(s, \hat{h})\right)$ are compact wherever they exist, any possible singularities of $\hat{T}(s)$ and $\hat{T}_{J M}(s)$ are poles [6, 9]. Since $N_{J M}(s, \hat{h})$ has an inverse for Re $s>-\gamma_{J M}$, then, if $\tilde{N}_{J M}(s, \hat{h})$ has an inverse in $\operatorname{Re} s>-\gamma_{J M}^{\prime}$, it follows that the operator-valued function $N(s, \hat{h})$ has an inverse in $\operatorname{Re} s>\operatorname{Min}\left(-\gamma_{J M},-\gamma_{J M}^{\prime}\right)$ by construction of $N(s, \hat{h}) . \hat{T}(s)$ has a pole with $\operatorname{Re} s_{0}>\operatorname{Min}\left(-\gamma_{J M},-\gamma_{J M}^{\prime}\right)$ if and only if 
the operator-valued function $N(s, \hat{h})$ has an eigenvalue one at $s=s_{0}$ so that $N_{J M}^{-1}\left(s_{0}, \hat{h}\right)$, $\tilde{N}_{J M}\left(s_{0}, \hat{h}\right)$, and $N\left(s_{0}, \hat{h}\right)$ are not full rank. Define the vector function of delays $f(\hat{h}):=$ $\operatorname{Sup}\left(\operatorname{Re} s: \tilde{N}_{J M}(s, \hat{h})\right.$ has an eigenvalue one). This function is continuous on its definition domain $\mathbb{R}_{+}^{0}$. Since the delay-free system (2.1) is g.u.e.s., then $f(0)<0$ so that (2.1) is not (is) g.u.e.s.i.d. if and only if $f\left(\hat{h}_{0}\right)>0$ for some vector of delays $\hat{h}_{0}$ with $h_{0, m+1}=0$ and $h_{0, i}>0$ for $i \neq m+1$ (if and only if $f(\hat{h})<0$ for all $\hat{h}$ with $h_{0, m+1}=0$ and $h_{0, i}>0$ for $\left.i \neq m+1\right)$. Furthermore, there is a domain properly included in $\mathbb{R}_{+}^{0}$ such that $\hat{\tilde{T}}_{J M}^{\prime}\left(j \omega, \varphi_{0}\right)$ has an eigenvalue one if $f\left(\hat{h}_{0}\right)=0$ since $\tilde{N}_{J M}\left(j \omega \hat{h}_{0}\right)$ is not full rank for some real $\omega$, where $\varphi_{0 i}=\omega h_{0 i}\left(i=1,2, \ldots, m+m^{\prime}+1\right)$ with $h_{0, m+1}=0$. But then, from the definition of $\hat{T}^{\prime}\left(j \omega, \varphi_{0}\right),[-\pi, \pi]$ always exists such that the ranks of $\hat{T}^{\prime}\left(j \omega, \varphi_{0}\right)$ and $\hat{T}_{J M}^{\prime}\left(j \omega, \varphi_{0}\right)$ are less than $n$ and the result has been proved. Note that the test for negative $\omega$ is unnecessary since eventual complex poles appear in conjugate pairs.

Theorem 3.1 may be used in particular for the special cases of Section 2 as follows.

Corollaries. Assume, in the following corollaries, that $\dot{x}(t)=L x_{t}$ is g.u.e.s. for $\hat{h}=0$ for all $\phi \in C_{e}(h)$.

COROLLARY 3.2 (delay-free dynamics). If $M$ is strictly Hurwitzian, then $\dot{x}(t)=L x_{t}$ is g.u.e.s. for all $\hat{h} \in[0, \infty)$, that is, g.u.e.s.i.d. if and only if the operator-valued function (3.1) exists, where

$$
\begin{aligned}
& \hat{T}_{J M}^{\prime}(j \omega)=(j \omega I-M)^{-1} \\
\Delta \hat{T}_{J M}^{\prime}(j \omega, \varphi)= & M-A+\sum_{i=1}^{m} A_{i} e^{j \varphi_{1 i}} \\
& +j \omega\left(\sum_{i=0}^{m^{\prime}} \hat{\alpha}_{i}(j \omega) A_{\alpha_{i}} e^{j \varphi_{2 i}}+\sum_{i=m^{\prime}+1}^{m^{\prime}+m^{\prime \prime}} \hat{\alpha}_{i}(j \omega) A_{\alpha_{i}}\left(1-e^{j \varphi_{3 i}}\right)\right) \\
& \times\left(\sum_{i=0}^{m^{\prime}} \alpha_{i}(0) A_{\alpha_{i}} e^{j \varphi_{2 i}}+\sum_{i=m^{\prime}+1}^{m^{\prime}+m^{\prime \prime}} \alpha_{i}(0) A_{\alpha_{i}}\left(1-e^{j \varphi_{3 i}}\right)\right) .
\end{aligned}
$$

Corollary 3.3 (delay-free and point-delayed dynamics). If $\dot{z}(t)=L_{J M} z_{t} \equiv M z(t)+$ $\sum_{i=1}^{m} A_{i} z\left(t-h_{i}\right)$ is g.u.e.s.i.d. for some given real square $n$-matrix $M$, then $\dot{x}(t)=L x_{t}$ is g.u.e.s.i.d. if and only if (3.1) exists, where

$$
\begin{gathered}
\hat{T}_{J M}^{\prime}(j \omega, \varphi)=\left[j \omega I-M-\sum_{i=1}^{m} A_{i} e^{j \varphi_{1 i}}\right]^{-1}, \\
\Delta \hat{T}_{J M}^{\prime}(j \omega, \varphi)= \\
\times-A+j \omega\left(\sum_{i=0}^{m^{\prime}} \hat{\alpha}_{i}(j \omega) A_{\alpha_{i}} e^{j \varphi_{2 i}}+\sum_{i=m^{\prime}+1}^{m^{\prime}+m^{\prime \prime}} \hat{\alpha}_{i}(j \omega) A_{\alpha_{i}}\left(1-e^{j \varphi_{3 i}}\right)\right) \\
\times\left(\sum_{i=0}^{m^{\prime}} \alpha_{i}(0) A_{\alpha_{i}} e^{j \varphi_{2 i}}+\sum_{i=m^{\prime}+1}^{m^{\prime}+m^{\prime \prime}} \alpha_{i}(0) A_{\alpha_{i}}\left(1-e^{j \varphi_{3 i}}\right)\right) .
\end{gathered}
$$

COROLlaRY 3.4 (delay-free and convolution Volterra-type dynamics). If $\dot{z}(t)=L_{J M} z_{t}$ $\equiv M z(t)+\int_{0}^{t} d \alpha(\tau) A_{\alpha} z(t-\tau)$ is g.u.e.s. for all bounded $z(0) \in \mathbb{R}^{n}$ for some given real 
square $n$-matrix $M$, then $\dot{x}(t)=L x_{t}$ is g.u.e.s.i.d. if and only if (3.1) exists, where

$$
\begin{aligned}
& \hat{T}_{J M}^{\prime}(j \omega, \varphi)=\left[j \omega\left(I-\hat{\alpha}(j \omega) A_{\alpha}\right)-M+\alpha(0) A_{\alpha}\right]^{-1} \\
& \Delta \hat{T}_{J M}^{\prime}(j \omega, \varphi)= M-A+\sum_{i=1}^{m} A_{i} e^{j \varphi_{1 i}} \\
&+j \omega\left(\sum_{i=1}^{m^{\prime}} \hat{\alpha}_{i}(j \omega) A_{\alpha_{i}} e^{j \varphi_{2 i}}+\sum_{i=m^{\prime}+1}^{m^{\prime}+m^{\prime \prime}} \hat{\alpha}_{i}(j \omega) A_{\alpha_{i}}\left(1-e^{j \varphi_{3 i}}\right)\right) \\
& \times\left(\sum_{i=1}^{m^{\prime}} \alpha_{i}(0) A_{\alpha_{i}} e^{j \varphi_{2 i}}+\sum_{i=m^{\prime}+1}^{m^{\prime}+m^{\prime \prime}} \alpha_{i}(0) A_{\alpha_{i}}\left(1-e^{j \varphi_{3 i}}\right)\right) .
\end{aligned}
$$

COROLLARY 3.5 (delay-free and infinitely-distributed delayed dynamics). If $\dot{z}(t)=$ $L_{J M} z_{t} \equiv M z(t)+\sum_{i=0}^{m^{\prime}} \int_{0}^{t} d \alpha_{i}(\tau) A_{\alpha_{i}} z\left(t-\tau-h_{i}^{\prime}\right)$ is g.u.e.s. for all bounded $\phi \in C_{e}(h)$ for some given real square $n$-matrix $M$, then $\dot{x}(t)=L x_{t}$ is g.u.e.s.i.d. if and only if (3.1) exists, where

$$
\begin{aligned}
\hat{T}_{J M}^{\prime}(j \omega, \varphi)= & {\left[j \omega\left(I-\sum_{i=0}^{m^{\prime}} \hat{\alpha}_{i}(j \omega) A_{\alpha_{i}} e^{j \varphi_{2 i}}\right)-M\right]^{-1}, } \\
\Delta \hat{T}_{J M}^{\prime}(j \omega, \varphi)= & M-A+\sum_{i=1}^{m} A_{i} e^{j \varphi_{1 i}}+j \omega\left(\sum_{i=m^{\prime}+1}^{m^{\prime}+m^{\prime \prime}} \hat{\alpha}_{i}(j \omega) A_{\alpha_{i}}\left(1-e^{j \varphi_{3 i}}\right)\right) \\
& \times\left(\sum_{i=m^{\prime}+1}^{m^{\prime}+m^{\prime \prime}} \alpha_{i}(0) A_{\alpha_{i}}\left(1-e^{j \varphi_{3 i}}\right)\right) .
\end{aligned}
$$

COROLLARY 3.6 (delay-free and finitely-distributed delayed dynamics). If $\dot{z}(t)=$ $L_{J M} z_{t} \equiv M z(t)+\sum_{i=m^{\prime}+1}^{m^{\prime}+m_{t-h_{i}^{\prime \prime}}^{\prime}} \int_{\alpha_{i}}(t-\tau) A_{\alpha_{i}} z(\tau)$ is g.u.e.s. for all bounded $\phi \in C_{e}(h)$ for some given real square $n$-matrix $M$, then $\dot{x}(t)=L x_{t}$ is g.u.e.s.i.d. for all bounded $\phi \in C_{e}(h)$ if and only if (3.1) exists, where

$$
\begin{aligned}
\hat{T}_{J M}^{\prime}(j \omega, \varphi)= & {\left[j \omega\left(I-\sum_{i=m^{\prime}+1}^{m^{\prime}+m^{\prime \prime}} \hat{\alpha}_{i}(j \omega) A_{\alpha_{i}}\left(1-e^{j \varphi_{3 i}}\right)\right)-M\right]^{-1}, } \\
\Delta \hat{T}_{J M}^{\prime}(j \omega, \varphi)= & M-A+\sum_{i=1}^{m} A_{i} e^{j \varphi_{1 i}}+j \omega\left(\sum_{i=0}^{m^{\prime}} \hat{\alpha}_{i}(j \omega) A_{\alpha_{i}} e^{j \varphi_{2 i}}\right) \\
& \times\left(\sum_{i=0}^{m^{\prime}} \alpha_{i}(0) A_{\alpha_{i}} e^{j \varphi_{2 i}}+\sum_{i=m^{\prime}+1}^{m^{\prime}+m^{\prime \prime}} \alpha_{i}(0) A_{\alpha_{i}}\left(1-e^{j \varphi_{3 i}}\right)\right) .
\end{aligned}
$$

The global uniform exponential stability of (2.1) may be investigated provided that each group of delayed dynamics (like, for instance, all point delays, infinitely distributed delays, or finitely distributed ones) is successively introduced in the system as addressed as follows. Note, for instance, that the system with combined delay-free and point-delayed dynamics $\dot{z}(t)=L_{J M} z_{t} \equiv A z(t)+\sum_{i=1}^{m} A_{i} z\left(t-h_{i}\right)$ is g.u.e.s.i.d. for all bounded $\phi \in C_{e}(h)$ if and only if $\left(I-(j \omega I-A)^{-1} \sum_{i=1}^{m} A_{i} e^{j \varphi_{1 i}}\right)^{-1}$ exists for all $\omega \in(0, \infty)$ and all $\varphi_{1 i} \in[-\pi, \pi], i=1,2, \ldots, m$, provided that $A$ is strictly Hurwitzian (i.e., if the undelayed auxiliary system is g.u.e.s. so that Corollary 3.2 holds). 
COROLlary 3.7 (delay-free, point-delayed, and infinitely distributed-delayed dynamics). If $\dot{z}(t)=L_{J M} z_{t} \equiv A z(t)+\sum_{i=1}^{m} A_{i} z\left(t-h_{i}\right)+\sum_{i=0}^{m^{\prime}} \int_{0}^{t} d \alpha_{i}(\tau) A_{\alpha_{i}} z\left(t-\tau-h_{i}^{\prime}\right)$ is g.u.e.s.i.d. for all bounded $\phi \in C_{e}(h)$ if and only if

$$
\left[I-(j \omega I-A)^{-1}\left(\sum_{i=1}^{m} A_{i} e^{j \varphi_{1 i}}+\sum_{i=0}^{m^{\prime}}\left(j \omega \hat{\alpha}_{i}(j \omega)-\alpha_{i}(0)\right) e^{j \varphi_{2 i}}\right)\right]^{-1}
$$

exists for all $\omega \in(0, \infty)$ and all $\varphi_{k i} \in[-\pi, \pi], k_{i} \in N_{i}, i=1,2$, provided that $A$ is strictly Hurwitzian (i.e., provided that the undelayed auxiliary system is g.u.e.s. so that Corollary 3.2 holds with $M=A$ ), it is also true that $\dot{z}(t)=L_{J M} z_{t}$ is g.u.e.s.i.d. for all bounded $\phi \in C_{e}(h)$ if and only if

$$
\left(I-\left(j \omega I-A-\sum_{i=1}^{m} A_{i} e^{j \varphi_{1 i}}\right)^{-1}\right)\left[\sum_{i=0}^{m^{\prime}}\left(j \omega \hat{\alpha}_{i}(j \omega)-\alpha_{i}(0)\right) e^{j \varphi_{2 i}}\right]^{-1}
$$

exists for all $\omega \in(0, \infty), \varphi_{21}=0$, and all $\varphi_{k i} \in[-\pi, \pi], k_{i} \in N_{i}, i=1,2$, provided that A is strictly Hurwitzian (i.e., provided that the auxiliary system with both undelayed and point delayed dynamics is g.u.e.s. so that Corollary 3.3 holds with $M=A$ ).

We might proceed in that way by giving conditions that ensure that each added group of delays maintains the uniform stability independent of delay provided that it was g.u.e.s.i.d. before adding those delays. It is also interesting to derive conditions for losing or ensuring uniform stability dependent on delay as follows.

THEOREM 3.8. Assume that (2.1) is g.u.e.s. for $\hat{h}=0$. Thus, $\dot{x}(t)=L x_{t}$ is not (resp., is) g.u.e.s. for all sets of delays that satisfy simultaneously $h_{i}=\varphi_{1 i} / \omega, h_{k}^{\prime}=\varphi_{2 k} / \omega$, and $h_{\ell}^{\prime}=\varphi_{3 k} / \omega$ with $\varphi_{21}=0$ for some $\omega \in \mathbb{R}_{+}, \varphi_{i k} \in[-\pi, \pi], i=1,2, \ldots, m, k=$ $1,2, \ldots, m^{\prime}$, and $l=m^{\prime}+1, m^{\prime}+2, \ldots, m^{\prime}+m^{\prime \prime}$ provided that (3.1) does not exist (resp., exists).

Proof (outline). The proof follows directly since for such sets of delays, the proof of Theorem 3.1 fails since there is some pole of $\hat{T}(s)$, so that it is not holomorphic, on $\operatorname{Re} s \geq 0$ since $\hat{T}^{\prime}(j \omega, \varphi)$ does not have an inverse for some $\omega \in \mathbb{R}_{+}$and $\varphi=$ $\left(\varphi_{1}^{T}, \varphi_{2}^{T}, \varphi_{3}^{T}\right)^{T}$ of components in the real interval $[-\pi, \pi]$.

REMARK 3.9. Note that in Theorem 3.8 the rank of an the operator-valued function (3.1) has to be tested in order to ensure the existence of its inverse within an appropriate stability domain. If the auxiliary system is not g.u.e.s.i.d., the test directly fails. On the other hand, since the eigenvalues of the operator-valued function are continuous functions of the arguments and since such a function is continuously differentiable with respect to its arguments, the implicit function theorem ensures that if the test does not fail at a set of delays (or constants $h_{(\cdot)}^{\prime}$ characterizing distributed delays), it does not fail either within open neighborhoods of such delays (or constants). Thus, the system is g.u.e.s for delays in some open neighborhoods of the $h_{(\cdot)}$ - and $h_{(\cdot)}^{\prime}$-constants where the system is g.u.e.s. 
4. Uniform stability under impulsive forcing terms. The stability under impulsive forcing terms in (2.1) may be formulated under a direct extension of the basic results of Section 3 as follows.

THEOREM 4.1. Assume that $\dot{x}(t)=L x_{t}$ is g.u.e.s.i.d., which holds if Theorem 3.1 holds with $\hat{T}^{\prime}(j \omega, \varphi)=\left[I-\hat{T}_{J M}^{\prime}(j \omega, \varphi) \Delta \hat{T}_{J M}^{\prime}(j \omega, \varphi)\right]^{-1} \hat{T}_{J M}^{\prime}(j \omega, \varphi)$ existing within some appropriate domain with $\hat{T}_{J M}^{\prime}(j \omega, \varphi)$ defining any g.u.e.s.i.d. auxiliary system defined for some $J$-triple, and, thus, $\Delta \hat{T}_{J M}^{\prime}(j \omega, \varphi)=\hat{T}_{J M}^{\prime-1}(j \omega, \varphi)-\hat{T}^{\prime-1}(j \omega, \varphi)$. Assume also that the forcing impulsive vector function $v:[0, \infty) \rightarrow \mathbb{R}^{n}$ satisfies $\left|b_{i}\right| \leq K_{i} e^{-i \rho}$ with $t_{i+1}-t_{i} \geq$ $T_{\min } \geq\left(\rho-\rho^{\prime}\right) / \gamma$, some real constant $\rho^{\prime} \in(0, \rho)$, and $K_{i} \in \mathbb{R}_{+}$being bounded constants for all $i \in \mathbf{I}$. Thus, the solution of (2.1), $x(t, \phi)$, is bounded on $\mathbb{R}_{+}$and $x(t, \phi) \rightarrow 0$ exponentially as $t \rightarrow \infty$ for any $\phi \in C_{e}(h)$.

Proof. Let $x_{0}(t, \phi)$ be the unique solution of the homogeneous $\dot{x}(t)=L x_{t}$ for $t \geq 0$ for any given $\phi \in C_{e}(h)$. Thus, the unique solution $x(t, \phi)$ for $t \geq 0$ for identical $\phi \in$ $C_{e}(h)$ of the forced $\dot{x}(t)=L x_{t}+u(t)$, with $v(t)=\sum_{i \in \mathbf{I}} b_{i} e^{-\left(t-t_{i}\right)}$, is bounded on $\mathbb{R}_{+}$and satisfies

$$
\left\|x(t, \phi)-x_{0}(t, \phi)\right\| \leq\left\|\sum_{i \in \mathbf{I}} T\left(t-t_{i}\right) b_{i}\right\| \leq\left|\sum_{i \in \mathbf{I}} K e^{-\gamma\left(t-t_{i}\right)} b_{i}\right|
$$

since $\|T(t)\| \leq K e^{-\gamma t}\left(\gamma \in \mathbb{R}_{+}\right)$. If $\operatorname{Card}(\mathbf{I})<\infty$, the $x(t)$ is bounded and $x(t) \rightarrow 0$ exponentially as $t \rightarrow \infty$ if $x_{0}(t) \rightarrow 0$ exponentially as $t \rightarrow \infty$. It only remains to consider the case when $\operatorname{Card}(\mathbf{I})=\infty$. Since $\left|b_{i}\right| \leq K_{i} e^{-i \rho}, \gamma t_{i}-i \rho \geq-i \rho^{\prime}$ for some real $\rho^{\prime} \in(0, \rho)$ since $t_{i}=\sum_{k=1}^{i} T_{k} \geq i T_{\min } \geq i\left(\rho-\rho^{\prime}\right) / \gamma$ with $T_{i} \geq T_{\min }=\left(\rho-\rho^{\prime}\right) / \gamma$, for all $i \in \mathbf{I}$. Thus, since $\sum_{i=0}^{\infty} e^{-i \rho^{\prime}}<\infty$,

$$
\begin{aligned}
\left\|x\left(t_{k}, \phi\right)-x_{0}\left(t_{k}, \phi\right)\right\| & \leq K \bar{K} \sum_{i=0}^{k} e^{-i \rho^{\prime}} e^{-\gamma\left(t_{k}-t_{i}\right)} \\
& \leq K \bar{K} \sum_{i=0}^{\infty} e^{-\gamma t_{k}} e^{-i\left(\rho^{\prime}-\rho\right)} \leq K \bar{K} e^{-\gamma t_{k}} \frac{\varepsilon}{1-\varepsilon} \longrightarrow 0
\end{aligned}
$$

as $t_{k} \rightarrow \infty$ and $x \in L_{\infty}\left([0, \infty) ; \mathbb{R}^{n}\right)$, with $\varepsilon=e^{-\left(\rho-\rho^{\prime}\right)}<1$ for all $\phi \in L_{\infty}\left([0, \infty) ; \mathbb{R}^{n}\right)$. Then, it is also exponentially continuous over I. Since the solution $x(t, \phi)$ of (2.1) is continuous over the finite intervals of nonzero measures $\left[t_{k}, t_{k+1}\right), k \in \mathbf{I}$, it cannot diverge within such intervals. Thus, $x(t, \phi)$ is bounded and converges exponentially to zero as $t \rightarrow \infty$.

REMARK 4.2. Note that $x\left(t_{i}^{+}\right)-x\left(t_{i}^{-}\right)=b_{i}$ with $b_{i}$ vanishing exponentially as $i \rightarrow \infty$ in Theorem 4.1. Assume that at discontinuity points the solution trajectory satisfies $x\left(t_{i}^{+}\right)=\left(I+B_{i}\right)\left\|x\left(t_{i}^{-}\right)\right\|$with $B_{i} \in \mathbb{R}^{n \times n}$ for all $i \in \mathbf{I}$; that is, $b_{i}=B_{i} x\left(t_{i}^{-}\right)$for $i \in \mathbf{I}$. Thus, $\left\|x\left(t_{i}^{+}, \phi\right)-x\left(t_{i}^{-}, \phi\right)\right\| \leq \operatorname{Sup}_{i \in \mathbf{I}}\left(\left\|B_{i}\right\|\right)\left\|x\left(t_{i}^{-}, \phi\right)\right\| \leq \bar{K}\left\|x\left(t_{i}^{-}, \phi\right)\right\|$, where $0 \leq \bar{K}=$ $\operatorname{Sup}_{i \in \mathbf{I}}\left(\left\|B_{i}^{-}\right\|\right)<\infty$; that is, the variation function at the discontinuity points of the trajectory is bounded and the increments converge asymptotically to zero provided that $\left\|x\left(t_{i}^{-}, \phi\right)\right\| \rightarrow 0$ as $t_{i} \rightarrow \infty$. 
THEOREM 4.3. Assume that the homogeneous system (2.1) is g.u.e.s.i.d. for all $\phi \in$ $C_{e}(h)$. Thus, it is g.u.e.s.i.d. for all $\phi \in C_{e}(h)$ and any impulsive $v(t)=\sum_{i \in \mathbf{I}} b_{i} \delta\left(t-t_{i}\right)$ with Card(I) being finite or infinite if any of the subsequent conditions holds for all $i\left(\geq i_{0}\right) \in \mathbf{I}$, some arbitrary $i_{0} \in \mathbb{N}$ :

(i) $\operatorname{Min}_{i \in \mathbf{I}}\left(t_{\ell+1}-t_{\ell}\right) \geq T_{\min }>(1 / i \gamma) \sum_{k=1}^{i} \ln \left(\left\|I+B_{k}\right\|\right)$;

(ii) if $T_{0}=t_{1}$ and $T_{i}=t_{i+1}-t_{i}$, for all $i \in \mathbf{I}$, then $\sum_{k=1}^{i} T_{k} \geq \sum_{k=1}^{i} \ln \left(\left\|I+B_{k}\right\|\right)^{1 / \gamma}$;

(iii) $T_{i} \geq \ln \left(\left\|I+B_{i}\right\|\right)^{1 / \gamma}$ for all $i=i_{0}, i_{0}+1, \ldots, \operatorname{Card}(\mathbf{I})-1$.

Proof. Let $T_{i}$ be $T_{i}=t_{i+1}-t_{i}$, for all $i \in \mathbf{I}$, and $\tau \in\left[0, T_{i}\right)$. Thus, from (2.1),

$$
\begin{aligned}
& x\left(t_{i+1}+\tau, \phi\right)=T(\tau) x\left(t_{i}^{+}\right)+\int_{-h}^{0} T\left(\tau-\tau^{\prime}\right) x\left(t_{i+1}+\tau, \phi\right) d \tau^{\prime} \\
& x\left(t_{i}^{+}, \phi\right)=\left(I+B_{i}\right) x\left(t_{i}^{-}, \phi\right) \\
&=\left(I+B_{i}\right)\left[T\left(t_{i}\right) \phi\left(0^{+}\right)+\sum_{k=1}^{i-1} T\left(t_{i}-t_{k}\right) B_{k} x\left(t_{k}^{-}, \phi\right)\right] \\
&=\left(I+B_{i}\right)\left[T\left(t_{i}-t_{i-1}\right) x\left(t_{i-1}^{+}, \phi\right)\right. \\
&\left.\quad+\int_{-h}^{0} T\left(t_{i}-t_{i-1}-\tau^{\prime}\right) x\left(t_{i-1}+\tau^{\prime}, \phi\right) d \tau^{\prime}\right] .
\end{aligned}
$$

Taking Euclidean norms in the above relations, one gets

$$
\begin{aligned}
\left\|x\left(t_{i}^{+}, \phi\right)\right\| \leq & \frac{\gamma+1-e^{-\gamma h}}{\gamma}\left\|\left(I+B_{i}\right) T\left(t_{i}-t_{i-1}\right)\right\| \operatorname{Sup}_{0 \leq \tau \leq t_{i}^{-}}(\|x(\tau, \phi)\|) \\
\leq & K e^{-\gamma\left(t_{i}-t_{i-1}\right)}\left\|I+B_{i}\right\| \operatorname{Sup}_{t_{i-1-h}^{-} \leq \tau \leq t_{i}^{-}}(\|x(\tau, \phi)\|), \\
\left\|x\left(t_{i}+\tau, \phi\right)\right\| \leq & \|T(\tau)\|\left[\left\|x\left(t_{i}^{+}, \phi\right)\right\|+\int_{-h}^{0}\left\|R\left(\tau^{\prime}\right)\right\|\left\|x\left(t_{i}+\tau^{\prime}, \phi\right)\right\| d \tau^{\prime}\right] \\
& \times \frac{\gamma+1-e^{-\gamma h}}{\gamma}\|T(\tau)\| \operatorname{Sup}_{t_{i}^{+}+\tau-h \leq \tau^{\prime} \leq t_{i+1}^{+}}\left(\left\|x\left(\tau^{\prime}, \phi\right)\right\|\right) \\
\leq & K e^{-\gamma t} \operatorname{Sup}_{t_{i}^{+}+\tau-h \leq \tau^{\prime} \leq t_{i+1}^{+}}\left(\left\|x\left(\tau^{\prime}, \phi\right)\right\|\right)
\end{aligned}
$$

with $R:[0, \infty) \rightarrow \mathbb{R}^{n \times n}$ being a matrix function that defines the factored representation $T(t-\tau)=T(t) R(\tau)$ so that $\|R(\tau)\| \leq e^{-\gamma \tau}$ since $\|T(t-\tau)\| \leq K e^{-\gamma(t-\tau)}$ for all $t \geq \tau \geq 0$. Since $\int_{-h}^{0}\|R(\tau)\| d \tau \leq\left(1-e^{-\gamma h}\right) / \gamma$, then

$$
\begin{gathered}
\left\|x\left(t_{i}^{+}, \phi\right)+\int_{-h}^{0} R\left(\tau^{\prime}\right) x\left(t_{i}^{+}+\tau^{\prime}, \phi\right) d \tau^{\prime}\right\| \\
\quad \leq\left\|x\left(t_{i}^{+}, \phi\right)\right\|+\frac{1}{\gamma} \operatorname{Sup}_{t_{i}^{-}-h \leq \tau \leq t_{i}^{+}}(\|x(\tau, \phi)\|) \\
\quad \leq \frac{\gamma+1-e^{-\gamma h}}{\gamma} \operatorname{Sup}_{t_{i}^{-}-h \leq \tau \leq t_{i}^{+}}(\|x(\tau, \phi)\|) .
\end{gathered}
$$


The recursive use of (4.5) for all $i \in \mathbf{I}$ while relating $x\left(t_{1}, \phi\right)$ to initial conditions $\phi$ : $[-h, 0] \rightarrow \mathbb{R}^{n}$ of supreme norm $\bar{\phi}$ leads to

$$
\begin{aligned}
\operatorname{Sup}_{t_{i}^{+} \leq \tau \leq t_{i+1}^{-}}(\|x(\tau, \phi)\|) & \leq \frac{\gamma+1-e^{-\gamma h}}{\gamma}\|T(\tau)\| \prod_{k=1}^{i}\left(\left\|I+B_{k}\right\|\right)\left\|T\left(t_{k}-t_{i+1}\right)\right\| \bar{\phi} \\
& \leq K e^{-\gamma\left(\tau+t_{i}\right)} \prod_{k=1}^{i}\left(\left\|I+B_{k}\right\|\right) \bar{\phi} .
\end{aligned}
$$

A sufficient condition for global uniform exponential stability independent of delay, after excluding any finite number of consecutive impulses in (2.1), what is irrelevant for stability analysis, is $e^{-i \gamma T_{\min }} \prod_{k=1}^{i}\left(\left\|I+B_{k+\ell}\right\|\right)<1$ for any finite integer $i \geq 0$ provided that $t_{i+1}-t_{i} \geq T_{\min }$ for any integer $i \geq \ell$. Thus, it follows that (2.1) is g.u.e.s.i.d. under (i) by taking logarithms in the above inequality. It is proved that (2.1) is g.u.e.s.i.d. under (ii) by replacing $e^{-i \gamma T_{\min }}$ by $e^{-t_{i}}=e^{-\sum_{k=1}^{i-1} T_{k}}$. The fact that (2.1) is g.u.e.s.i.d. under (iii) is direct since the fulfilment of (iii) guarantees that of (ii).

\section{Closed-loop uniform exponential stability under linear feedback}

5.1. Simple stability tests and stabilization of the closed-loop system. In the subsequent study, consider the unforced system (2.1). The discussion is limited to the case of delay-free combined point-delayed dynamics in (2.1); that is, $m^{\prime}=m^{\prime \prime}=0$. The extension to the general case is direct. The auxiliary system is $\dot{z}(t)=M z(t)$, that is, $J_{i}=\varnothing$ ( $i=1,2,3)$, with $M$ strictly Hurwitzian. Thus, (2.1) is g.u.e.s.i.d. From Corollary 3.2, (2.1) is g.u.e.s.i.d. if and only if

$$
\hat{T}(j \omega)=\left(I-(j \omega I-M)^{-1}\left[M-A+\sum_{i=1}^{m} A_{i} x\left(t-h_{i}\right)\right]\right)^{-1}(j \omega I-M)^{-1}
$$

exists for all $\omega \in \mathbb{R}_{+}$g.u.e.s. for $\hat{h}=0$, that is, for any bounded $x(0)=\phi(0) \in \mathbb{R}^{n}$. Note that $(j \omega I-M)^{-1}$ exists for all $\omega \in \mathbb{R}_{+}$since $M$ is strictly Hurwitzian. Consider the set $H_{\infty}(X)=\left\{x: \mathbb{C}_{+}^{0} \rightarrow X: \operatorname{Sup}_{\operatorname{Re} s>0}(\|x(s)\|)<\infty\right\}$, where $\mathbb{C}_{+}^{0}$ is the complex open righthand, side half-plane. A similar $H_{\infty}$ space is defined for the set of linear operators on $X$ by replacing $X \rightarrow L(X, X)$. Note that $\hat{T} \in H_{\infty}(L(X, X))$ where it exists. Simple calculations for $H_{\infty}$-norms yield

$$
\begin{aligned}
\gamma_{M} & :=\left\|(j \omega I-M)^{-1}\right\|_{\infty} \\
& =\operatorname{Max}\left\{\gamma \in \mathbb{R}_{+}: H_{M}:=\left[\begin{array}{cc}
M & \frac{I}{\gamma_{M}^{2}} \\
-I & -M^{T}
\end{array}\right] \text { has an eigenvalue on the imaginary axis }\right\} .
\end{aligned}
$$

$\hat{T}(j \omega)$ exists for all $\omega \in \mathbb{R}_{+}^{0}$ if

$$
1>\gamma_{M}\left[\|M-A\|_{2}+\sum_{i=1}^{m}\left\|A_{i}\right\|_{2}\right]
$$


since $\operatorname{Sup}_{\omega \in \mathbb{R}_{+}^{0}}\left\{\left\|M-A+\sum_{i=1}^{m} A_{i} e^{-j \omega h_{i}}\right\|_{2}\right\} \leq\|M-A\|_{2}+\sum_{i=1}^{m}\left\|A_{i}\right\|_{2}$, where $\mathbb{R}_{+}^{0}:=\mathbb{R}_{+} \cup\{0\}$ and $\|\cdot\|_{2}$ denotes the $l_{2}$-matrix norm for each $\omega \in \mathbb{R}_{+}$. Now consider the following feedback system subject to $m$ internal and $m^{\prime}$ external (denoted in the sequel as $h_{i}^{\prime}$ ) point delays:

$$
\dot{x}(t)=A x(t)+\sum_{i=1}^{m} A_{i} x\left(t-h_{i}\right)+B u(t)+\sum_{i=1}^{m^{\prime}} B_{i} u\left(t-h_{i}^{\prime}\right)
$$

with $B, B_{i} \in \mathbb{R}^{q \times n}\left(i=1,2, \ldots, m^{\prime}\right)$, where the control function $u:[0, \infty) \rightarrow \mathbb{R}^{q}$ is continuous and has range $U$, that is, $u \in C^{(0)}([0, \infty) ; U)$ while being generated from the control law:

$$
u(t)=K C x(t)+\sum_{i=1}^{m} K_{i} C x\left(t-h_{i}\right)+\sum_{i=1}^{m^{\prime}} K_{i}^{\prime} u\left(t-h_{i}^{\prime}\right)
$$

with real matrices $K, K_{i} \in \mathbb{R}^{q \times r}, C \in \mathbb{R}^{r \times n}$. It is assumed that $y$ is an $r$-measurable output signal $y:[0, \infty) \rightarrow \mathbb{R}^{r}$ defined by $y(t)=C x(t)$ for all $t \geq 0$. Taking Laplace transforms in (5.4) with zero initial conditions with $s=j \omega$, one directly gets the closedloop relations

$$
\begin{aligned}
\left(j \omega I-A-\sum_{i=1}^{m} A_{i} e^{-j \omega h_{i}}\right) \hat{x}(j \omega) & =B_{0}+\sum_{i=1}^{m^{\prime}} B_{i} e^{-j \omega h_{i}^{\prime}} \hat{u}(j \omega), \\
\left(I-\sum_{i=1}^{m} K_{i}^{\prime} e^{-j \omega h_{i}^{\prime}}\right) \hat{u}(j \omega) & =\sum_{i=0}^{m} K_{i} C e^{-j \omega h_{i}} \hat{x}(j \omega) .
\end{aligned}
$$

The substitution of (5.7) into (5.6) yields $\hat{S}_{\mathcal{C}}(j \omega) \hat{x}(j \omega)=0$ with

$$
\begin{gathered}
\hat{S}_{\mathcal{C}}(j \omega)=(j \omega I-M) \hat{T}_{c}(j \omega), \\
\hat{T}_{C}(j \omega)=I-(j \omega I-M)^{-1}\left\{\begin{array}{l}
M-A-B K C+\sum_{i=1}^{m}\left(A_{i}+B K_{i} C\right) e^{-j \omega h_{i}} \\
\left.+\left(\sum_{i=1}^{m^{\prime}} B_{i} e^{-j \omega h_{i}^{\prime}}\right) S_{u}^{-1}(j \omega)\left(K C+\sum_{i=1}^{m} K_{i} C e^{-j \omega h_{i}}\right)\right\}
\end{array}\right.
\end{gathered}
$$

with $S_{u}(j \omega)=I-\sum_{i=1}^{m^{\prime}} K_{i}^{\prime} e^{-j h_{i}^{\prime} \omega}$. The closed-loop system is g.u.e.s.i.d. if $\hat{T}_{c}^{-1}(j \omega)$ exists provided that $S_{u}^{-1}(j \omega)$ exists for all $\omega \in \mathbb{R}_{+}$. The following result holds.

THEOREM 5.1. The closed-loop system is g.u.e.s.i.d. if the following conditions are fulfilled:

(a) $M$ is strictly Hurwitzian so that the auxiliary system $\dot{z}(t)=M z(t)$ is g.u.e.s.;

(b) $\sum_{i=1}^{m^{\prime}}\left\|K_{i}^{\prime}\right\|<1$;

(c) the closed-loop system is g.u.e.s. in the absence of delays, that is, for $h_{i}=0, h_{k}^{\prime}$ $\left(i=1,2, \ldots, m, k=1,2, \ldots, m^{\prime}\right)$; 
(d)

$$
\begin{aligned}
1>\gamma_{M}\left\{\|M-A-B K C\|_{2}+\sum_{i=1}^{m}\left\|A_{i}+B K_{i} C\right\|_{2}\right. \\
\left.\quad+\sum_{i=1}^{m^{\prime}}\left\|B_{i}\right\|_{2} \sum_{i=1}^{m^{\prime}}\left\|K_{i}\right\|_{2}\|C\|_{2} \frac{1}{1-\left\|\sum_{i=1}^{m^{\prime}}\right\| K_{i}^{\prime}\left\|_{2}\right\|}\right\} .
\end{aligned}
$$

The subsequent cases are of interest to address Theorem 5.1.

CASE A ( $C=I$ (i.e., linear state feedback) and $(A, B)$ is a completely controllable pair). Thus, the eigenvalues of $(A+B K C)$ and then those of $(M-A-B K C)$ may be prefixed to arbitrary positions in $\operatorname{Re} s<0$ (see, e.g., $[3,7,13])$ and then any norm of $(M-A-B K C)$ may be made as small as required. Furthermore, $\gamma_{M}$ may be as small as suitable to fulfil (5.9) for any sets of controller gains $K_{(\cdot)}$ and $K_{(\cdot)}^{\prime}$ that fulfil $\left|\sum_{i=1}^{m^{\prime}} K_{i}^{\prime}\right|_{2}<1$. Assume, for instance, that the eigenvalues of $(A+B K C)$ are chosen identical to those of $M$ located at $\operatorname{Re} s \leq-\rho<0$. Assume also that $B$ and $C$ are full column and row rank, respectively. Thus, $\gamma_{M} \leq 1 / \rho^{n}$ if $K$ is chosen as follows:

$$
\begin{aligned}
K & =\left[\left(B \otimes C^{T}\right)^{T}\left(B \otimes C^{T}\right)\right]^{-1}\left(B \otimes C^{T}\right)^{T}\left(m_{v}-a_{v}\right) \\
& =\left(B^{T} B \otimes C C^{T}\right)^{-1}\left(B^{T} \otimes C\right)\left(m_{v}-a_{v}\right) \\
& =\left(\left(B^{T} B\right)^{-1} \otimes\left(C C^{T}\right)^{-1}\right)\left(B^{T} \otimes C\right)\left(m_{v}-a_{v}\right),
\end{aligned}
$$

where $P \otimes Q=\left(p_{i j} Q\right)$ is the direct Kronecker product of the $a \times b$ and $c \times d$ matrices $P$ and $Q$, respectively, and

$$
m_{v}=\operatorname{Vec}(M)=\operatorname{col}\left(\begin{array}{c}
m_{1} \\
m_{2} \\
\vdots \\
m_{n}
\end{array}\right), \quad a_{v}=\operatorname{Vec}(A)=\operatorname{col}\left(\begin{array}{c}
a_{1} \\
a_{2} \\
\vdots \\
a_{n}
\end{array}\right) \text {, }
$$

where $m_{v}$ and $a_{v}$ are column vectors formed with the consecutive rows $m_{(\cdot)}^{T}$ of $M$ and $a_{(.)}^{T}$ of $A$, respectively, written in order with ordered entries. It has been used that $(P \otimes Q)^{T}=P^{T} \otimes Q^{T}$ and that $B^{T} B$ and $C C^{T}$ are both nonsingular since $B$ and $C$ are full column and row rank, respectively.

CASE B $\left(C=I,(A, B)\right.$ is stabilizable (but not controllable), and $\left(A_{i}, B\right)$ are completely controllable for $i=1,2, \ldots, m)$. Thus, a finite gain $\gamma_{A+B K}=\left\|(s I-A-B K)^{-1}\right\|_{\infty}$ can be designed for each given $A$-matrix but it cannot be prefixed. Thus, the controller gain matrix $K$ may be chosen so that the controllable and observable modes of $(A+B K)$ are arbitrarily close to those of $M[3,7]$. Also, the controller gains $K_{i}$ may be calculated so 
that $\left(A_{i}+B_{i} K_{i}\right)$ are zero $(i=1,2, \ldots, m)$ and $K_{i}^{\prime}$ such that $\left\|K_{i}^{\prime}\right\|$ are arbitrarily small for $i=1,2, \ldots, m^{\prime}$. Thus, condition (d) of Theorem 5.1 may be fulfilled for any finite $\gamma_{A+B K}$ (i.e., for any $K$ that stabilizes the stabilizable pair $(A, B)$ ).

CASE C $(C \neq I$ (i.e., output feedback is used) and the triple $(A, B, C)$ is controllable and observable with $\operatorname{rank}(B)=q, \operatorname{rank}(C)=r$, and $\max (q, r) \geq n$ ). Thus, the eigenvalues of $(A+B K C)$ may be prefixed to positions being arbitrarily close to prescribed ones inside the closed left-half complex plane, and any norm of $(M-A-B K C)$ may be made as small as convenient for design purposes. Also, $\gamma_{A+B K C}$ may be made arbitrarily small, and the design to accomplish with (see, in particular, (5.7)) may be performed similarly as in Case A.

CASE $\mathbf{D}(C \neq I$ (i.e., output feedback is used) and the triple $(A, B, C)$ is stabilizable and detectable with $\operatorname{rank}(B)=q, \operatorname{rank}(C)=r$, and $\max (q, r) \geq n)$. Furthermore, $\left(A_{i}, B, C\right)$ is controllable and observable $(i=1,2, \ldots, m)$. The design may be performed as in Case C.

REMARK 5.2. Once The global uniform exponential stability of the inhomogeneous closed-loop system has been achieved, then it may be guaranteed to be g.u.e.s. under impulsive forcing signals by establishing additional conditions as in Theorems 4.1 and 4.3. The above analysis dictates that the absence or presence of controller external delays is irrelevant for design purposes. Note, in particular, that condition (c) of Theorem 5.1 is satisfied directly if the related controller gains are zeroed.

The extensions of the above results in this section to the presence of distributed delays are not difficult. Assume, for instance, that the state (or only the output) is available for measurement, that is, $C=I$ (or $C \neq I$ ), and that there are distributed delays in the system. Thus, the control law (5.7) may be generalized to

$$
\begin{aligned}
u(t)= & K C x(t)+\sum_{i=1}^{m} K_{i} C x\left(t-h_{i}\right) \\
& +\sum_{i=0}^{m^{\prime}} \int_{0}^{t} d \alpha_{i}(\tau) K_{\alpha_{i}} x\left(t-\tau-h_{i}^{\prime}\right) \\
& +\sum_{i=m^{\prime}+1}^{m^{\prime}+m^{\prime \prime}} \int_{t-h_{i}^{\prime}}^{t} d \alpha_{i}(t-\tau) K_{\alpha_{i}} x(\tau) .
\end{aligned}
$$

Define $\beta=\operatorname{Max}_{0 \leq i \leq m^{\prime}+m^{\prime \prime}}\left(\beta_{i} \in \mathbb{R}_{+}\right.$such that $\left.\int_{0}^{\infty}\left|\alpha_{i}(\tau)\right| d \tau<\infty\right)$. Thus, $\hat{\alpha}_{i}(s)=$ $\int_{0}^{\infty} \alpha_{i}(\tau) e^{-s \tau} d \tau \leq \int_{0}^{\infty}\left|\alpha_{i}(\tau)\right| e^{\beta \tau} d \tau<\infty$ for $\operatorname{Re} s \geq-\beta\left(i=0,1, \ldots, m^{\prime}+m^{\prime \prime}\right)$; it follows that $\left|\alpha_{i}(t)\right| \leq K e^{(\varepsilon-\beta) t}$, for all $t \geq 0$ and any real constant $\varepsilon>0$, so that $\operatorname{Max}_{0 \leq i \leq m^{\prime}+m^{\prime \prime}}\left(\left|\hat{\alpha}_{i}(s)\right|\right) \leq K /|s+\beta-\varepsilon|<K /|s+\beta|$ for Re $s<-\gamma$. Then,

$$
\begin{aligned}
\left\|s \hat{\alpha}_{i}(s)-\alpha_{i}(0)\right\|_{\infty} & \leq K \operatorname{Sup}_{\omega \in \mathbb{R}_{+}^{0}}\left(\left|\frac{j \omega}{\sqrt{\beta^{2}+\omega^{2}}}\right|\right) \\
& =K+\operatorname{Max}_{0 \leq i \leq m^{\prime}+m^{\prime \prime}}\left(\left|\alpha_{i}(0)\right|\right) \leq 2 K .
\end{aligned}
$$


Thus, condition (d) of Theorem 5.1 becomes (after substituting (5.12) into (2.1), via (5.13), and obtaining a relation in Laplace transforms for the closed-loop system description)

$$
\begin{aligned}
1>\gamma_{M}\{ & {\left[\|M-A-B K C\|_{2}+\sum_{i=1}^{m}\left\|A_{i}+B K_{i} C\right\|_{2}\right] } \\
& \left.+2 k\left[\sum_{i=0}^{m^{\prime}}\left\|A_{\alpha_{i}}+B K_{\alpha_{i}} C\right\|_{2}+2 \sum_{i=m^{\prime}+1}^{m^{\prime}+m^{\prime \prime}}\left\|A_{\alpha_{i}}+B K_{\alpha_{i}} C\right\|_{2}\right]\right\} .
\end{aligned}
$$

Very similar considerations as for point-delays (Theorem 5.1) may be used for the case when $(A, B, C)$ is controllable and observable or for $(A, B)$ being controllable and $C=I$ with $r=n$ and for that when $(A, B, C)$ is stabilizable and detectable but the triples $\left(A_{i}, B, C\right)$ and $\left(A_{\alpha_{k}}, B, C\right)\left(i=1,2, \ldots, m, k=0,1, \ldots, m^{\prime}+m^{\prime \prime}\right)$ are controllable and observable, or, if $C=I$, the pairs $\left(A_{i}, B\right)$ and $\left(A_{\alpha_{k}}, B\right)\left(i=1,2, \ldots, m, k=0,1, \ldots, m^{\prime}+m^{\prime \prime}\right)$ are all controllable.

\section{Examples}

EXAMPLE 6.1 (the simple first-order system $\dot{x}(t)=-a x(t)+a_{1} x(t-h)$ with $x(0)=$ $\left.x_{0}\right)$. If $a>0$, then Theorem 5.1 yields $\gamma_{a}=\left\|(s-a)^{-1}\right\|_{\infty}=1 /|a|$ and the system is g.u.e.s.i.d. if $1>\gamma_{a}\left|a_{1}\right| \operatorname{Sup}_{\omega \in \mathbb{R}_{+}^{0}}\left(\left|e^{-j h \omega}\right|\right)=\gamma_{a}\left|a_{1}\right|$ provided that the auxiliary system $\dot{z}(t)=-a z(t)$ with $z(0)=z_{0}$ is g.u.e.s., that is, $a>0$. Thus, the system is g.u.e.s.i.d. if $a>\left|a_{1}\right|>0$. The same conclusion is obtained by applying Gronwall's lemma [12], as follows. Compute the solution to the system of differential equation to obtain

$$
\begin{aligned}
g(t):= & e^{a(t-h)} x(t, \phi) \\
= & e^{-a h} x_{0}+\int_{0}^{h} e^{a(\tau-h)} \phi(\tau-h) d \tau \\
& +\int_{h}^{t} e^{a(\tau-h)} a_{1} x(\tau-h, \phi) d \tau .
\end{aligned}
$$

Substituting $g(\tau-h)=e^{a(\tau-h)} x(\tau-h, \phi)$, taking modules, and upper-bounding the absolute value of the last integral by $\int_{0}^{t}\left|e^{a(\tau-h)} x(\tau-h, \phi)\right| d \tau$, one gets

$$
|g(t)| \leq e^{-a h}\left|x_{0}\right|+\int_{0}^{h}\left|e^{a(\tau-h)} \phi(\tau-h)\right| d \tau+\left|a_{1}\right| \int_{h}^{t}|g(\tau-h)| d \tau
$$

so that $\|x(t, \phi)\| \leq v(\phi) e^{-\left(a+\left\|a_{1}\right\|\right) t}$ for all $t \geq 0$, where $v=\left\|x_{0}\right\| e^{-a h}+\left|a_{1} / a\right| \mid e^{a h}-$ $1 \mid \operatorname{Sup}_{-h \leq \tau \leq 0}(\|\phi(\tau)\|)$. Thus, exponential stability follows for $a>\left|a_{1}\right|>0$. Assume, for instance, that $a<0$ so that the auxiliary system is unstable. Thus, use the delayfree control law $u(t)=k x(t)$ with $k>-a$. Thus, the above results hold by replacing $a \rightarrow k-|a|$ so that the closed-loop uniform exponential stability independent of delay is ensured if $k>|a|+\left|a_{1}\right|$ still from Theorem 5.1. Note that Theorem 3.1 holds with $\hat{T}(s)=\left(s+a-a_{1} e^{-h s}\right)^{-1}, \hat{T}_{J M}^{-1}=(s+a)^{-1}$, and $M=a$.

EXAMPLE 6.2. Consider the multiple point-delay $n$ th-order system under an impulsive forcing term $\dot{x}(t)=A x(t)+\sum_{i=1}^{m} A_{i} x(t-h)+\sum_{i \in \mathbf{I}} b_{i} \delta\left(t-t_{i}\right)$. Thus, the unique 
solution for any admissible $n$-vector real function $\phi$ of initial conditions is

$$
\begin{aligned}
x(t) & =e^{A\left(t-t_{i}\right)} x\left(t_{i}^{+}\right)+\sum_{i=1}^{m} \int_{t_{i}}^{t} e^{A(t-\tau)} A_{i} x\left(\tau-h_{i}\right) d \tau \\
& =T(t) \phi\left(0^{+}\right)+\int_{-h}^{0} T(t-\tau) \phi(\tau) d \tau+\sum_{i \in \mathbf{I}} T\left(t-t_{i}\right) U\left(t-t_{i}\right) b_{i}
\end{aligned}
$$

for $t \in\left(t_{i}, t_{i+1}\right), x\left(t_{i+1}^{+}\right)=x\left(t_{i+1}^{-}\right)+b_{i+1}$ with $h=\operatorname{Max}_{1 \leq i \leq m}\left(h_{i}\right)$ with $T(t)$ satisfying $\dot{T}(t)=A T(t)+\sum_{i=1}^{m} A_{i} T(t-h)$ with $T(0)=I$ and $T(t)=0$ for $t<0$.

Several situations are now discussed.

(a) Assume that the auxiliary system $\dot{z}(t)=A z(t)$ is uniformly exponentially stable for all bounded $z(0)$, that is, $A$ is strictly Hurwitzian with stability abscissa $-\vartheta=$ $\left(-\vartheta_{i}\right)<0$ and the associated dominant eigenvalue $\mu=\mu_{i}$. Assume also that there is no impulsive action, that is, all the $b_{i}$ are zero. Thus, from Theorem 5.1, the current delayed system is guaranteed to be g.u.e.s.i.d. if $|\mathcal{Y}|^{\mu}>\sum_{i=1}^{m}\left\|A_{i}\right\|_{2}$ with $\gamma_{A}^{-1}=|\mathcal{V}|^{\mu}$.

(b) If $A$ is not strictly Hurwitzian, then assume that a control $u(t)=K x(t)$ is applied through the control matrix $B$ with $(A, B)$ being controllable. Thus, the delay-free closedloop dynamics can be defined by the strictly Hurwitzian $n$-matrix $M=A+B K$ (which may be chosen as the delay-free dynamics of the auxiliary system) of the same stability abscissa and dominant eigenvalue multiplicity as above. Thus, the closed-loop system is g.u.e.s.i.d. if $|\vartheta|^{\mu}>\sum_{i=1}^{m}\left\|A_{i}\right\|_{2}$ for a controller gain matrix $K$ being an existing solution of $(B \otimes I) K_{v}=m_{v}-a_{v}$ with $K_{v}=\operatorname{Vec}(K), m_{v}=\operatorname{Vec}(M)$, and $a_{v}=\operatorname{Vec}(A)$.

(c) If $(A, B)$ is only stabilizable, then the stability abscissa is $\operatorname{Min}\left(-\vartheta,-9^{\prime}\right)<0$, where $(-\vartheta)<0$ is obtained from the relocated closed-loop controllable poles through the controller gain matrix $K$ and $\left(-\vartheta^{\prime}\right)<0$ is the stability abscissa of the uncontrollable openloop stable (since the system is stabilizable) poles which cannot be relocated through feedback. Thus, the delayed system is g.u.e.s.i.d. if $\operatorname{Max}\left(\left.\left|\vartheta^{\mu},\right| \vartheta^{\prime}\right|^{\mu^{\prime}}\right)>\sum_{i=1}^{m}\left\|A_{i}\right\|_{2}$.

(d) If the state is not available for measurement but $(A, B, C)$ is controllable and observable or (at least) stabilizable and detectable for appropriate control and output matrices $B$ and $C$, respectively, then the closed-loop stabilization problem may be solved in light of Theorem 5.1.

(e) Now, assume that the impulsive input is nonzero. If there is a finite number of impulses, then the above conditions of uniform stability still remain valid. If there is an infinite number of impulses $b_{i}=B_{i} x\left(t_{i}^{-}\right)$, then the global uniform stability independent of delay is preserved if all the time intervals in-between two consecutive impulses satisfy the lower-bound constraint $T_{\min } \geq \operatorname{Sup}_{k \in \mathbf{I}}\left(1 / i \gamma^{\prime}\right) \sum_{k=1}^{i}\left\|I+B_{k}\right\|$ with $\gamma^{\prime}=\gamma_{A}^{-1}-\sum_{i=1}^{m}\left\|A_{i}\right\|_{2}$ from Theorems 4.3(ii) and 5.1 provided that $\gamma_{A}:=\left\|(s I-A)^{-1}\right\|_{\infty}>$ $\sum_{i=1}^{m}\left\|A_{i}\right\|_{2}$.

(f) If, in case (e), $A$ is not strictly Hurwitzian and a closed-loop stabilization is performed, then $\gamma_{A}$ is replaced by the appropriate gain $\gamma_{M}$ for $M=A+B K$. If all the $b_{i}$ $(i \in \mathbf{I})$ converge exponentially to zero while being state-independent, Theorem 4.3 may be used instead of Theorem 5.1.

(g) Now, assume that an auxiliary system $\dot{z}(t)=A z(t)+A_{1} z\left(t-h_{1}\right)$ is g.u.e.s.i.d. for all $\phi \in C_{e}\left(h_{1}\right)$ and any delay $h_{1}$. A sufficient condition is $\left|\vartheta_{A}\right|^{\mu_{A}}>\left\|A_{1}\right\|_{2}$ with $\gamma_{A}^{-1}=\left|\vartheta_{A}\right|^{\mu_{A}}$, where $\left(-\vartheta_{A}\right)<0$ is the stability abscissa of $A$ provided that $A$ is strictly 
Hurwitzian with the dominant eigenvalue being of multiplicity $\mu_{A}$. Define the $H_{\infty}$-norm

$$
\begin{aligned}
\gamma_{\text {aux }} & :=\left\|\left(s I-A-A_{1} e^{-h s}\right)^{-1}\right\|_{\infty} \\
& \leq\left\|(s I-A)^{-1}\right\|_{\infty} \cdot\left\|I-(s I-A)^{-1} A_{1} e^{-h s}\right\|_{\infty} \leq \frac{\gamma_{A}}{1-\gamma_{A}\left\|A_{1}\right\|_{2}}
\end{aligned}
$$

provided that $\left\|A_{1}\right\|_{2}<\gamma_{A}^{-1}=\left.\left|\vartheta_{A}\right|\right|_{A}$. Then, a sufficient condition for the current system to be g.u.e.s.i.d. when no impulsive input is injected is that $1>\gamma_{\mathrm{aux}} \sum_{i=2}^{m}\left\|A_{i}\right\|_{2}$, which is guaranteed if $1>\gamma_{A} \sum_{i=2}^{m}\left\|A_{i}\right\|_{2} /\left(1-\gamma_{A}\left\|A_{1}\right\|_{2}\right)$ provided that $\left\|A_{1}\right\|_{2}<\gamma_{A}^{-1}=\left|\vartheta_{A}\right|^{\mu_{A}}$. If $A+A_{1} e^{-h s}$ has stable eigenvalues but $A$ is not strictly Hurwitzian, that is, $\dot{z}^{\prime}(t)=$ $A z^{\prime}(t)$ is not g.u.e.s.i.d., then $\gamma_{\text {aux }}$ is finite but it cannot be calculated from sufficiencytype conditions for stability using (6.4). However, the system is still g.u.e.s.i.d. if $1>$ $\gamma_{\text {aux }} \sum_{i=2}^{m}\left\|A_{i}\right\|_{2}$.

(h) Now, assume that in case (g) there is an impulsive input as in case (e) consisting of infinitely many impulses. Thus, the current system is g.u.e.s.i.d. if the impulses occur at consecutive times being not less than $\operatorname{Sup}_{k \in \mathbf{I}}\left(1 / i \gamma_{\text {aux }}^{\prime}\right) \sum_{k=1}^{i}\left\|I+B_{k}\right\|$, except possibly on a set of zero measure, with $\gamma^{\prime-1} \rightarrow \gamma^{\prime-1}$ aux $=\left(1-\gamma_{A}\left\|A_{1}\right\|_{2}\right) / \gamma_{A}-\sum_{i=2}^{m}\left\|A_{i}\right\|_{2}$, provided that $\left\|A_{1}\right\|_{2}<\gamma_{A}^{-1}$ and $\sum_{i=2}^{m}\left\|A_{i}\right\|_{2}<\gamma_{A}^{-1}\left(1-\gamma_{A}\left\|A_{1}\right\|_{2}\right)$, from Theorems 4.3(ii) and 5.1.

EXAMPLE 6.3. Consider the second-order scalar functional equation $\ddot{x}(t)=-a \dot{x}(t)+$ $b x(t-h)$. The equation is decomposed into two first-order equations as follows:

$$
\begin{gathered}
x(t)=x_{1}(t), \quad \dot{x}_{1}(t)=x_{2}(t), \\
\dot{x_{2}}(t)=-a x_{2}(t)+b x_{1}(t)-\int_{-h}^{0} b x_{2}(t+\tau) d \tau .
\end{gathered}
$$

Consider the Lyapunov functional candidate

$$
V\left(x_{1 t}, x_{2 t}\right)=x_{2 t}^{2}-b x_{1 t}^{2}+\xi \int_{-h}^{0} \int_{t+s}^{t} x_{2 t}^{2}(\tau) d \tau d s
$$

(see [4]), whose time-derivative is

$$
\begin{aligned}
\dot{V}\left(x_{1 t}, x_{2 t}\right)= & -2 a x_{2}^{2}(t)-\int_{-h}^{0} 2 b x_{2}(\tau) x_{2}(t+\tau) d \tau \\
& +\xi \int_{-h}^{0} \int_{t+s}^{t}\left[x_{2}^{2}(t)-x_{2}^{2}(t+\tau)\right] d \tau \\
\leq & \int_{-h}^{0}\left\{\left[-\left(\frac{2 a}{r}\right)-b+\xi\right]\right\} x_{2}^{2}(s)+(-b-\xi) x_{2}^{2}(t+s) d s .
\end{aligned}
$$

Thus, the system is globally asymptotically stable, dependent on delay if $(-2(a / h)+$ $\xi-b)<0$ for some real constant $\xi>0$ if $x_{i}(t)$, for $i=1$ or 2 , is nonzero for some subinterval of nonzero measure of $[t-h, t]$, any $t \geq t_{0}$ (some finite $t_{0} \in R_{+}^{0}$ ), or, equivalently, $\operatorname{Max}(0,-b)<\xi<2(a / h)+b$. This holds for all $h>0$ if $\operatorname{Min}(a, b) \geq 0$ and $a$ and $b$ are not simultaneously zero. A general necessary condition for a given $h$ is that $(a / h+b)>0$ or, equivalently, $h>-a / b$. Note also that a necessary condition for exponential stability for $h=0$ is that $a>0$ and $b<0$, which follows from the Routh-Hurwitz criterion. As a result, if $a>0$ and $b<0$, then the system is globally uniformly asymptotically 
stable if $h \in[0, a /|b|)$. Theorem 4.3 may be applied as follows. Decompose the system of second-order functional differential equations into two first-order differential equations as follows:

$$
\dot{x}(t)=A x(t)+A_{1}(x(t)-x(t-h)),
$$

where

$$
x(t)=\left(x_{1}(t), x_{2}(t)\right)^{T}, \quad A=\left[\begin{array}{cc}
0 & 1 \\
b & -a
\end{array}\right], \quad A_{1}=\left[\begin{array}{cc}
0 & 0 \\
-b & 0
\end{array}\right]
$$

The stability abscissa of $A$ is $(-a)$ if $a<2|b|$ or $-\left(a-\sqrt{a^{2}-4|b|}\right)$ otherwise. Thus, the system is g.u.e.s.i.d. if $a>0, b<0$, and $a>2|b|+\sqrt{a^{2}-4|b|}$ since the stability condition for $a \geq 2|b|$ is $\left(a-\sqrt{a^{2}-4 b}\right)>2|b|$ (Theorem 5.1), and for $a<2|b|$, Theorem 5.1 fails in (6.4).

7. Conclusions. This paper has dealt with the global uniform exponential stability independent of delay (g.u.e.s.i.d.) of a class of homogeneous time-delay systems being possibly subject to combined point and distributed delays as well as integrodifferential Volterra-type delayed dynamics. The global stability is investigated for any real function of initial conditions being everywhere continuous on its definition domain, a real interval $[-h, 0]$, where $h$ is the maximum delay in the system, except possibly on a set of zero measure where the function of initial conditions possesses bounded discontinuities. Necessary and sufficient global uniform stability independent of delay conditions has been obtained if the delay-free system is globally uniformly exponentially stable (g.u.e.s.) and an auxiliary system is g.u.e.s.i.d. The obtained results have then been applied to a number of particular cases of interest by setting different auxiliary systems including the standard delay-free one. Furthermore, some extensions have been given for the case when the system is forced by impulsive inputs consisting of either a finite number of impulses or infinitely many impulses. It has been assumed either that the impulse amplitudes vanish exponentially or that the time interval between two inputs exceeds a prescribed threshold of sufficiently large length. Some extensions have been given by considering the closed-loop stabilization of time-delay systems of the given class. Finally, some illustrative examples have also been presented.

ACKNOWLEDGMENT. The authors are very grateful to the Spanish Ministry of Education and to the University of Basque Country for their partial support of this work through Research Grants DPI 2000-0244 and 1/UPV/EHU 00I06.I06-EB 8235/2000, respectively.

\section{REFERENCES}

[1] N. U. Ahmed, Optimal control of infinite-dimensional systems governed by integrodifferential equations, Differential Equations, Dynamical Systems, and Control Science, Lecture Notes in Pure and Appl. Math., vol. 152, Dekker, New York, 1994, pp. 383402. 
[2] C. F. Alastruey, M. de la Sen, and J. R. González de Mendívil, The stabilizability of integrodifferential systems with two distributed delays, Math. Comput. Modelling 21 (1995), no. 8, 85-94.

[3] S. Barnett, Polynomials and Linear Control Systems, Monographs and Textbooks in Pure and Applied Mathematics, vol. 77, Marcel Dekker, New York, 1983.

[4] T. A. Burton, Stability and Periodic Solutions of Ordinary and Functional-Differential Equations, Mathematics in Science and Engineering, vol. 178, Academic Press, Florida, 1985.

[5] G. Da Prato and M. Iannelli, Linear integro-differential equations in Banach spaces, Rend. Sem. Mat. Univ. Padova 62 (1980), 207-219.

[6] R. F. Datko, Time-delayed perturbations and robust stability, Differential Equations, Dynamical Systems, and Control Science, Lecture Notes in Pure and Appl. Math., vol. 152, Dekker, New York, 1994, pp. 457-468.

[7] M. de la Sen, An algebraic method for pole placement in multivariable systems with internal and external point delays by using single rate or multirate sampling, Dynam. Control 10 (2000), no. 1, 5-31.

[8] M. de la Sen and Ningsu Luo, Discretization and FIR filtering of continuous linear systems with internal and external point delays, Internat. J. Control 60 (1994), no. 6, 12231246.

[9] N. Dunford and J. T. Schwartz, Linear Operators. I. General Theory, Pure and Applied Mathematics, vol. 7, Interscience Publishers, New York, 1958.

[10] Y. Jiang and Y. Jurang, Positive solutions and asymptotic behavior of delay differential equations with nonlinear impulses, J. Math. Anal. Appl. 207 (1997), no. 2, 388-396.

[11] M. R. S. Kulenović, G. Ladas, and A. Meimaridou, Stability of solutions of linear delay differential equations, Proc. Amer. Math. Soc. 100 (1987), no. 3, 433-441.

[12] V. Lakshmikantham, D. D. Bănov, and P. S. Simeonov, Theory of Impulsive Differential Equations, Series in Modern Applied Mathematics, vol. 6, World Scientific Publishing, New Jersey, 1989.

[13] T. Sengadir, Asymptotic stability of nonlinear functional-differential equations, Nonlinear Anal. 28 (1997), no. 12, 1997-2003.

M. de la Sen: Departamento de Ingeniería de Sistemas y Automática, Instituto de Investigación y Desarrollo de Procesos (IIDP), Facultad de Ciencias, Universidad del País Vasco, Leioa (Bizkaia), Aptdo. 644 de Bilbao, 48080 Bilbao, Spain

E-mail address: wepdepam@1g.ehu.es

Ningsu Luo: Departamento de Electronica, Informatica y Automática, Escuela Politecnica Superior, Universidad de Girona, Campus Montilivi, Edificio P4, 17071 Girona, Spain

E-mail address: ningsu@eia.udg.es 


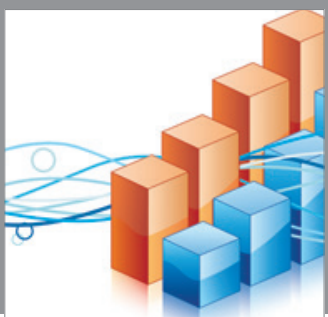

Advances in

Operations Research

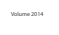

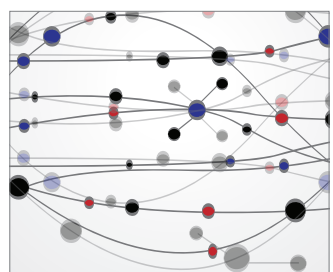

\section{The Scientific} World Journal
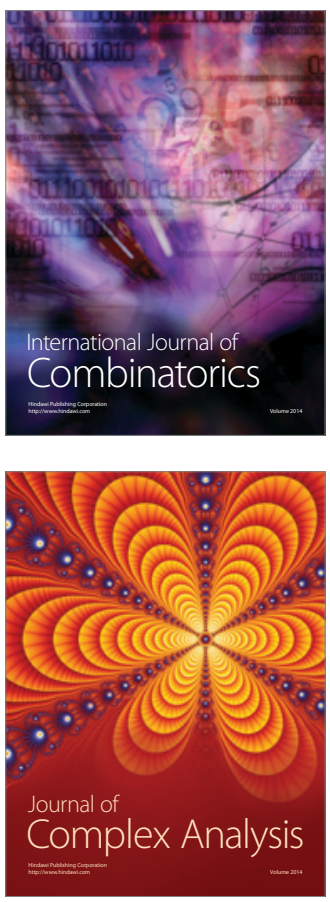

International Journal of

Mathematics and

Mathematical

Sciences
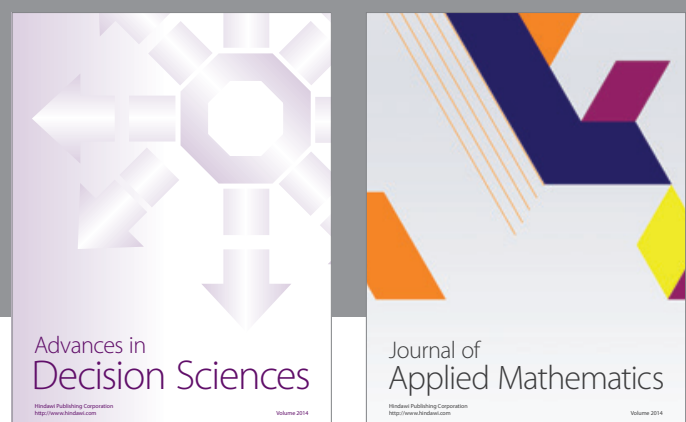

Journal of

Applied Mathematics
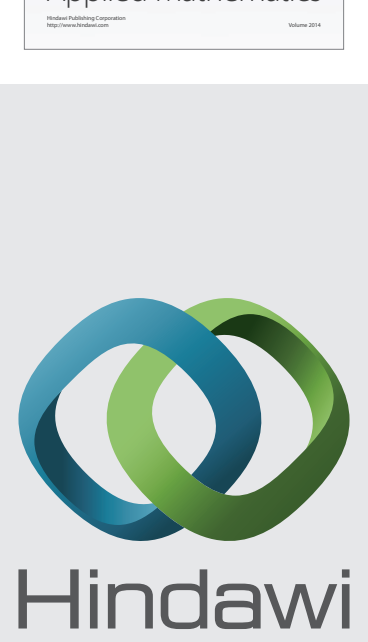

Submit your manuscripts at http://www.hindawi.com
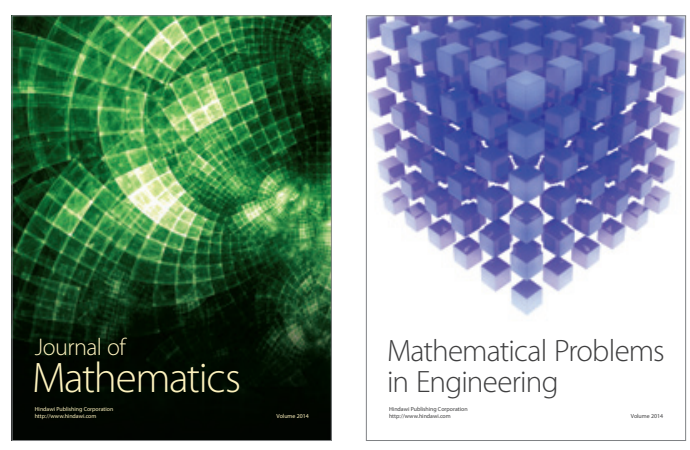

Mathematical Problems in Engineering
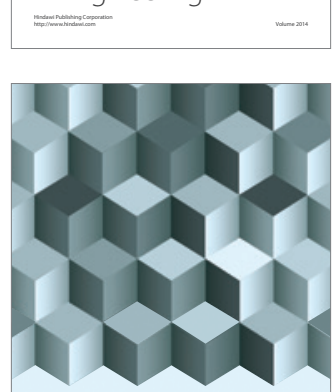

Journal of

Function Spaces
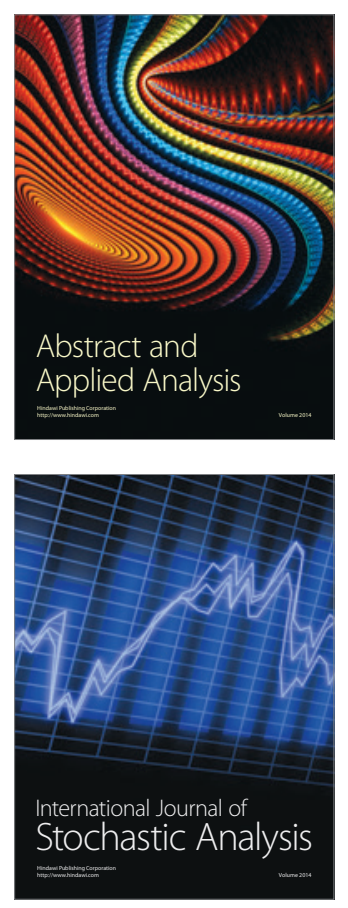

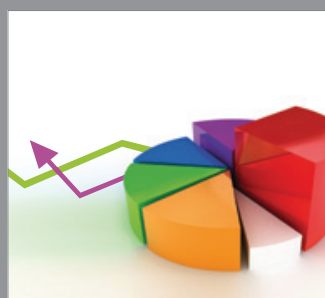

ournal of

Probability and Statistics

Promensencen
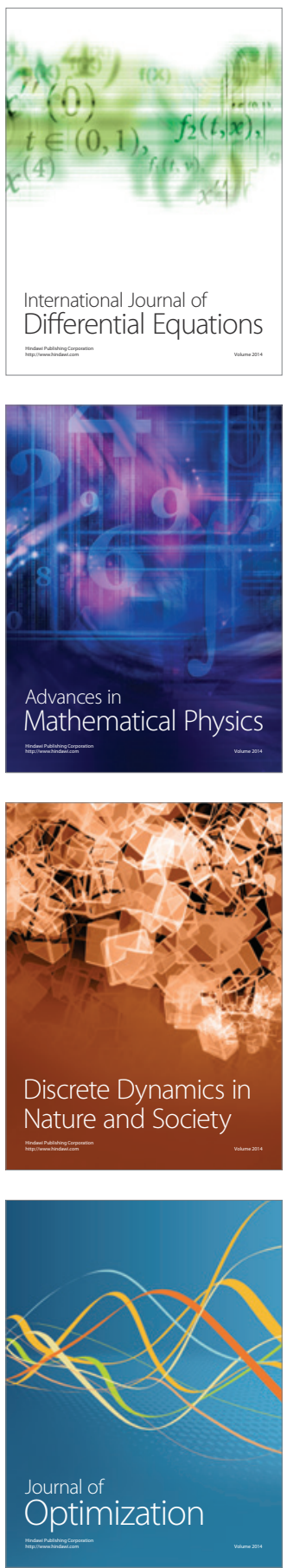